\title{
Electron beam dump constraints on light bosons with lepton flavor violating couplings
}

Takeshi Araki, ${ }^{a}$ Kento Asai ${ }^{b}$ and Takashi Shimomura ${ }^{c, d}$

${ }^{a}$ Faculty of Dentistry, Ohu University, 31-1 Sankakudo, Tomita-machi, Koriyama, Fukushima 963-8611, Japan

${ }^{b}$ Department of Physics, Faculty of Science, Saitama University,

Sakura-ku, Saitama 338-8570, Japan

${ }^{c}$ Faculty of Education, University of Miyazaki, 1-1 Gakuen-Kibanadai-Nishi, Miyazaki 889-2192, Japan

${ }^{d}$ Department of Physics, Kyushu University, 744 Motooka, Nishi-ku, Fukuoka 819-0395, Japan

E-mail: t-araki@den.ohu-u.ac.jp, asai@krishna.th.phy.saitama-u.ac.jp, shimomura@cc.miyazaki-u.ac.jp

ABSTRACT: We study constraints on light and feebly interacting bosons having charged lepton flavor violating couplings from electron beam dump experiments. Scalar, pseudoscalar, vector, and dipole type interactions of the bosons are analyzed, and excluded regions from the searches for decays into electron-positron pairs are derived. It is found that parameter regions unconstrained by flavor violating decays of muon can be excluded using the results of the E137 experiment. We also discuss the impact of the search for flavor violating decays of the light bosons in electron beam dump experiments.

Keywords: Beyond Standard Model, Gauge Symmetry, Higgs Physics

ArXIV EPRINT: 2107.07487 


\section{Contents}

1 Introduction 1

2 Interaction Lagrangian 3

2.1 Scalar interactions in two Higgs doublet model 3

2.2 Pseudoscalar interactions in axion-like-particle model 4

2.3 Vector interactions in $L_{\mu}-L_{\tau}$ model 4

2.4 Dipole CLFV in dark photon model 5

3 Light boson production in beam dump experiments $\quad 6$

$\begin{array}{lll}3.1 & \text { Differential cross section of bremsstrahlung process } & 7\end{array}$

$\begin{array}{ll}3.2 & \text { Number of signal events } \\ \end{array}$

4 Electron beam dump constraints $\quad 11$

$\begin{array}{lll}5 & \text { Flavor violating decay signal } & 14\end{array}$

$\begin{array}{lll}6 & \text { Summary } & 16\end{array}$

$\begin{array}{ll}\text { A Effective photon flux } & 17\end{array}$

$\begin{array}{ll}\text { B Function } \tilde{U}_{n}^{\ell} & 17\end{array}$

$\begin{array}{lr}\text { C Widths of CLFV decays of muons } & 18\end{array}$

\section{Introduction}

For the last decade, the searches for new physics beyond the Standard Model (SM) have been primarily led by the LHC experiment with particular attention to a parameter region of $\mathrm{TeV}$-scale mass with $\mathcal{O}(1)$ coupling. Despite a great deal of experimental and theoretical efforts, no signals of the new physics have been found so far. Given the fact, nowadays, there is a growing interest in neutral bosons having sub-GeV mass and feeble interactions with the SM particles. In fact, there are several theoretical motivations to consider such feebly-interacting light bosons. For instance, it plays a crucial role in the muon $g-2$ anomaly [1-3], the dark matter problem [4-11], and the Hubble tension [12-15]. Moreover, it was found that the existence of the light boson helps us understand the observed energy spectrum of high energy cosmic neutrino [16-24].

Feebly-interacting light bosons are expected to be long-lived and leave displaced vertex signals at experiments. Among possible experiments, beam dump experiments are especially suitable for searching such bosons. The bosons can be produced from an incoming 
beam on a target, travel long distance, and decay into an electron-positron pair near the detector distant from the target. In the 1980s, several beam dump experiments were carried out to search for light neutral scalar bosons, such as axions, by using proton beam [25] and electron beam [26-30]. These experimental constraints were later translated to those on light gauge bosons in refs. [31] and [32, 33]. At present, including the results of the beam dump experiments, the comprehensive analyses have been done for various types of light boson models, such as the dark photon [34, 35], $\mathrm{U}(1)_{B-L}$ gauge boson [34], $\mathrm{U}(1)_{L_{\alpha}-L_{\beta}}$ $(\alpha, \beta=e, \mu, \tau)$ gauge boson [34], dark Higgs boson [36], and axion-like-particles [37]. Also, in refs. [38-41], the prospect of a new beam dump experiment at the future ILC experiment is studied. Typically, beam dump experiments yield constraints in a mass region of $\mathrm{MeV}-\mathrm{GeV}$. On the other hand, below the MeV scale, there are strong constraints from astrophysics and cosmology [12, 42, 43]. As for the coupling constant, some regions below the beam dump constraints are excluded by the observation of supernovae [44, 45].

Although beam dump experiments are one of the best probes of the searches for feeblyinteracting light bosons, the constraints are derived only for minimal and simplest model setups, that is, only a single new particle with a new coupling constant is introduced. In the literature, however, several extensions have been proposed recently.

In this paper, given the current situation, we attempt to enlarge the availability of beam dump experiments for the extended scenarios. We are especially interested in extensions including Charged Lepton Flavor Violation (CLFV) [46-53]. As is well known, CLFV is one of the most evident signals for new physics beyond the SM. Many experimental searches have been carried out, and in most cases, CLFV decay processes of muons like $\mu \rightarrow e e e$ and $\mu \rightarrow e \gamma$ give the tightest upper bounds on CLFV couplings (see ref. [54] for a comprehensive review). On the other hand, in the sub-GeV mass region, electron beam dump experiments can possibly exclude a parameter region below the upper bounds from the CLFV decay processes of muons, and new bounds could be obtained. To illustrate the exclusion regions by electron beam dump experiments, we consider four types of interactions between the new light boson and charged leptons as benchmark models, i.e., Yukawa type interactions for a scalar boson, axion-like particle (ALP) type interactions for a pseudoscalar boson, and vector and dipole type interactions for a vector boson. We introduce CLFV couplings of electron $(e)$ and muon $(\mu)$, as well as Charged Lepton Flavor Conserving (CLFC) couplings, into the benchmark models. We then derive the exclusion regions of the CLFV couplings from the E137 experiment, which provides the most stringent constraints on the couplings with leptons.

This paper is organized as follows. In section 2, we introduce four types of CLFV interactions analyzed in this work. In section 3, the production cross sections of light bosons through bremsstrahlung processes and the formula to calculate the number of signal events are given. In section 4, the constraints on the light bosons with CLFV coupling by the E137 experiment are derived, and the results are compared with the existing bounds from the muon CLFV decays. In section 5, the impact of searches for flavor violating decays is discussed. Section 6 is devoted to summary. 


\section{Interaction Lagrangian}

We start our discussion by introducing interaction Lagrangians involving CLFV couplings analyzed in this work. As we explained in the introduction, we consider four types of CLFV interactions, i.e., Yukawa type interactions for a scalar boson, ALP type interactions for a pseudoscalar boson, and vector and dipole type interactions for a vector boson as benchmark models. We refer to these interactions as scalar, pseudoscalar, vector, and dipole interactions, respectively. In the following, we restrict our analyses only to CLFV in the $e \mu$ sector.

\subsection{Scalar interactions in two Higgs doublet model}

Firstly, we give the Yukawa type interaction Lagrangian. Such an interaction can be obtained in models with an extra leptophilic Higgs doublet scalar. The new Higgs doublet scalar is assumed to couple to only leptons and to contribute to the mass generation of the charged leptons. In this case, the mass matrix of the charged leptons consists of two parts originating from each Higgs doublet after developing vacuum expectation values (VEVs). In general, those two mass matrices are not necessarily diagonalized simultaneously, and the misalignment of the mass matrices generates CLFV interactions after the diagonalization. To avoid large contributions from the CLFV interaction by the SM Higgs boson, we assume the VEV of the extra Higgs doublet field to be much smaller than the electroweak scale. Then, the charged lepton masses are mostly determined by the mass matrix with the VEV of the SM Higgs boson. In such a case, the CLFV interactions are given from the Yukawa terms of the extra scalar boson.

The relevant interaction Lagrangian is given by

$$
\mathcal{L}_{\text {scalar }}=\sum_{\ell=e, \mu, \tau} y_{\ell} \overline{\ell_{L}} \phi \ell_{R}+y_{e \mu} \overline{e_{L}} \phi \mu_{R}+y_{\mu e} \overline{\mu_{L}} \phi e_{R}+\text { h.c. },
$$

where $\phi$ is the extra scalar boson, and $y_{\ell}$ and $y_{e \mu(\mu e)}$ are the CLFC and CLFV coupling constants, respectively. Here we omit the interactions obtained from the mixing between $\phi$ and the SM Higgs boson, due to the assumption of a small VEV for $\phi$.

From eq. (2.1), the total decay width of $\phi$ is given by

$$
\Gamma_{\text {tot }}=\sum_{\ell=e, \mu, \tau} \Gamma(\phi \rightarrow \ell \bar{\ell})+\Gamma(\phi \rightarrow e \bar{\mu})+\Gamma(\phi \rightarrow \mu \bar{e}) .
$$

Here the partial decay widths into the charged leptons are given by $[55,56]$

$$
\Gamma\left(\phi \rightarrow \ell \overline{\ell^{\prime}}\right)=\frac{1}{16 \pi} m_{\phi} \lambda\left(\frac{m_{\ell}^{2}}{m_{\phi}^{2}}, \frac{m_{\ell^{\prime}}^{2}}{m_{\phi}^{2}}\right)\left[S_{1}\left(1-\frac{m_{\ell}^{2}+m_{\ell^{\prime}}^{2}}{m_{\phi}^{2}}\right)-4 S_{2} \frac{m_{\ell} m_{\ell^{\prime}}}{m_{\phi}^{2}}\right],
$$

where $m_{\phi}$ is a mass of $\phi, \lambda$ is the Kallen function defined as follows:

$$
\lambda(a, b)=\sqrt{1+a^{2}+b^{2}-2 a-2 b-2 a b},
$$

and $S_{1}=2\left|y_{\ell}\right|^{2}$ and $S_{2}=\operatorname{Re}\left(y_{\ell}^{2}\right)$ for the CLFC decays, while $S_{1}=\left|y_{e \mu}\right|^{2}+\left|y_{\mu e}\right|^{2}$ and $S_{2}=\operatorname{Re}\left(y_{e \mu} y_{\mu e}\right)$ for the CLFV decays. 


\subsection{Pseudoscalar interactions in axion-like-particle model}

Secondly, we give the ALP type interaction Lagrangian which consists of the dimension 5 operators involving the derivative of a pseudoscalar boson. ALP is a pseudo-NambuGoldstone boson that appears from spontaneously breaking of a global symmetry. This kind of new particle is highly motivated in various contexts, such as the strong CP problem [5759], dark matter [60-62], and flavor problem [63-67]. In general, ALPs can have CLFV couplings, and such possibilities have been studied in the context of the electron and muon anomalous magnetic moment and CLFV decays [51-53].

The relevant interaction Lagrangian is given by

$$
\mathcal{L}_{\text {pseudoscalar }}=\frac{\partial_{\mu} a}{\Lambda}\left\{\sum_{\ell=e, \mu, \tau} c_{\ell \ell} \bar{\ell} \gamma^{\mu} \gamma_{5} \ell+c_{e \mu} \bar{e} \gamma^{\mu} \gamma_{5} \mu+c_{\mu e} \bar{\mu} \gamma^{\mu} \gamma_{5} e\right\},
$$

where $a$ is the pseudoscalar boson, and $\Lambda$ is a cutoff or the decay constant of the ALP.

From eq. (2.5), the total decay width of $a$ is given by

$$
\Gamma_{\text {tot }}=\sum_{\ell=e, \mu, \tau} \Gamma(a \rightarrow \ell \bar{\ell})+\Gamma(a \rightarrow e \bar{\mu})+\Gamma(a \rightarrow \mu \bar{e}) .
$$

Here, the partial decay widths into the charged leptons are obtained as $[48,68]$

$$
\Gamma(a \rightarrow \ell \overline{\ell \prime})=\frac{|P|^{2}}{8 \pi \Lambda^{2}} m_{a} \lambda\left(\frac{m_{\ell}^{2}}{m_{a}^{2}}, \frac{m_{\ell^{\prime}}^{2}}{m_{a}^{2}}\right) \frac{m_{a}^{2}\left(m_{\ell}+m_{\ell^{\prime}}\right)^{2}-\left(m_{\ell}^{2}-m_{\ell^{\prime}}^{2}\right)^{2}}{m_{a}^{2}},
$$

where $m_{a}$ stands for the pseudoscalar mass, $P=c_{\ell \ell}$ or $c_{e \mu(\mu e)}$ for the CLFC or the CLFV decays, respectively, and $\lambda(a, b)$ is given in eq. (2.4).

\subsection{Vector interactions in $L_{\mu}-L_{\tau}$ model}

Thirdly, we give the vector-type interaction Lagrangian. The interactions of the charged leptons with a new vector boson can be obtained by extending the gauge sector of the SM. For minimality, we consider an extra $\mathrm{U}(1)$ gauge symmetry in this work. CLFV vector interactions can be generated at both tree and loop levels. At tree level, the following conditions should be satisfied: 1) the different gauge charge assignments among the charged lepton flavors and 2) the misalignment between the interaction and mass eigenstates of the charged leptons. Then, CLFV interactions appear in the gauge sector after diagonalizing the mass matrix of the charged leptons. It should be noticed that the above conditions imply that the extra U(1) symmetry must be flavor-dependent. Therefore flavor universal gauge symmetries, such as the $\mathrm{U}(1)_{B-L}$ gauge symmetry, cannot induce the CLFV interactions in this way. One of the viable examples is the $\mathrm{U}(1)_{L_{\mu}-L_{\tau}}$ gauge symmetry [69-71] with extra Higgs doublets. Under the $\mathrm{U}(1)_{L_{\mu}-L_{\tau}}$ gauge symmetry, the lepton flavors are differently charged: only the mu and tau leptons $(\tau)$ are charged as +1 and -1 , respectively. Moreover, if there exist extra Higgs doublets charged under the $\mathrm{U}(1)_{L_{\mu}-L_{\tau}}$ gauge symmetry, off-diagonal elements in the charged lepton mass matrix are induced [71]. At loop level, CLFV vector interactions will be induced through the CLFV scalar loop for massive gauge bosons. In this case, the gauge symmetry does not have to be flavor-dependent. In 
this work, we only study the CLFV vector interactions at the tree level. However, analyses for the loop-induced CLFV interactions are essentially the same and will be translated by replacing the couplings with loop-induced ones in our results.

For general discussions, we parametrize the CLFV coupling by $\theta$ which is the mixing angle between the electron and muon. The mass and flavor eigenstates are connected by this mixing angle. Then, the Lagrangian of the vector interactions in mass eigenstate is given by

$$
\begin{gathered}
\mathcal{L}_{\text {vector }}=g^{\prime} Z_{\rho}^{\prime}\left(s^{2} \bar{e} \gamma^{\rho} e+c^{2} \bar{\mu} \gamma^{\rho} \mu+s c \bar{\mu} \gamma^{\rho} e+s c \bar{e} \gamma^{\rho} \mu\right) \\
+g^{\prime} Z_{\rho}^{\prime}\left(-\bar{\tau} \gamma^{\rho} \tau+\overline{\nu_{\mu}} \gamma^{\rho} \nu_{\mu}-\overline{\nu_{\tau}} \gamma^{\rho} \nu_{\tau}\right),
\end{gathered}
$$

where $Z^{\prime}$ is the $\mathrm{U}(1)_{L_{\mu}-L_{\tau}}$ gauge boson, $g^{\prime}$ is the gauge coupling constant of the $\mathrm{U}(1)_{L_{\mu}-L_{\tau}}$ gauge symmetry, and $s(c)=\sin \theta(\cos \theta)$. Here $\nu_{\mu}$ and $\nu_{\tau}$ are left-handed muon and tau neutrinos. It should be noted that, in general, there also can exist interactions through the kinetic mixing. Such interactions conserve lepton flavor and are independent of the mixing angle. Then, the flavor conserving productions and decays of $Z^{\prime}$ are modified. Although analyses of such a situation will lead to more general constraints, the increase of the parameters will make the analyses complicated. For simplicity, we assume that contributions from the kinetic mixing are negligible, and omit the kinetic mixing throughout this paper.

Given the Lagrangian in eq. (2.8), the total decay width of $Z^{\prime}$ is obtained as

$$
\Gamma_{\text {total }}=\Gamma\left(Z^{\prime} \rightarrow \nu \bar{\nu}\right)+\sum_{\ell=e, \mu, \tau} \Gamma\left(Z^{\prime} \rightarrow \ell \bar{\ell}\right)+\Gamma\left(Z^{\prime} \rightarrow e \bar{\mu}\right)+\Gamma\left(Z^{\prime} \rightarrow \mu \bar{e}\right)
$$

where the partial decay width into the neutrinos is given by

$$
\Gamma\left(Z^{\prime} \rightarrow \nu \bar{\nu}\right)=\frac{g^{\prime 2}}{12 \pi} m_{Z^{\prime}},
$$

in the massless limit of neutrinos, ${ }^{1}$ with $m_{Z^{\prime}}$ being a mass of $Z^{\prime}$. The partial decay widths into the charged leptons are

$$
\begin{aligned}
\Gamma\left(Z^{\prime} \rightarrow \overline{\ell \ell^{\prime}}\right)= & \frac{V^{2}}{24 \pi} m_{Z^{\prime}} \lambda\left(\frac{m_{\ell}^{2}}{m_{Z^{\prime}}^{2}}, \frac{m_{\ell^{\prime}}^{2}}{m_{Z^{\prime}}^{2}}\right) \\
& \times\left[2-\frac{m_{\ell}^{2}-6 m_{\ell} m_{\ell^{\prime}}+m_{\ell^{\prime}}^{2}}{m_{Z^{\prime}}^{2}}-\frac{\left(m_{\ell}^{2}-m_{\ell^{\prime}}^{2}\right)^{2}}{m_{Z^{\prime}}^{4}}\right],
\end{aligned}
$$

where $V=g^{\prime} s^{2}, g^{\prime} c^{2}$, or -1 for the CLFC decays, while $V=g^{\prime} s c$ for the CLFV decays.

\subsection{Dipole CLFV in dark photon model}

Lastly, we give the dipole-type interaction Lagrangian. We again consider an extra U(1) gauge symmetry and assume that there are no interactions between the new gauge boson

\footnotetext{
${ }^{1}$ Here, we assumed neutrinos are Dirac particles. For Majorana neutrinos, the partial decay width is multiplied by $1 / 2$.
} 
$A^{\prime}$ and the SM particles at tree level, similar to the dark photon model with vanishing kinetic mixing. Even in this case, CLFV interactions, as well as CLFC interactions, can be induced at the loop level. For instance, suppose new complex scalar bosons charged under the extra gauge symmetry exist and couple to the SM charged leptons with new fermions. The interaction between $A^{\prime}$ and charged leptons can be generated at one-loop in which the new scalar bosons and fermions propagate. Here we consider the following dipole-type interactions:

$$
\mathcal{L}_{\text {dipole }}=\frac{1}{2} \sum_{\ell=e, \mu, \tau} \mu_{\ell} \bar{\ell} \sigma^{\rho \sigma} \ell A_{\rho \sigma}^{\prime}+\frac{\mu^{\prime}}{2}\left(\bar{\mu} \sigma^{\rho \sigma} e+\bar{e} \sigma^{\rho \sigma} \mu\right) A_{\rho \sigma}^{\prime},
$$

where $\mu_{\ell}$ and $\mu^{\prime}$ are CLFC and CLFV dipole couplings, respectively, and $A_{\rho \sigma}^{\prime}$ stands for the field strength of $A^{\prime}$. We assume that the dipole couplings are real. One may imagine that there should exist similar CLFV interactions in which external $A^{\prime}$ is replaced by photon, which are strictly constrained by the MEG [72] and BaBar [73] experiments. However, it will be possible to suppress such dangerous electromagnetic dipole operators when the new gauge boson has an interaction vertex with neutral CP-even and odd scalars since the same vertex does not exist for the photon. One of such examples is the so-called dark photon model with dark Higgs particles [74].

Given the Lagrangian in eq. (2.12), the total decay width of $A^{\prime}$ is given by

$$
\Gamma_{\text {total }}=\sum_{\ell=e, \mu, \tau} \Gamma\left(A^{\prime} \rightarrow \ell \bar{\ell}\right)+\Gamma\left(A^{\prime} \rightarrow e \bar{\mu}\right)+\Gamma\left(A^{\prime} \rightarrow \mu \bar{e}\right),
$$

where

$$
\begin{aligned}
\Gamma\left(A^{\prime} \rightarrow \ell \overline{\ell^{\prime}}\right)= & \frac{D^{2}}{12 \pi} m_{A^{\prime}}^{3} \lambda\left(\frac{m_{\ell}^{2}}{m_{A^{\prime}}^{2}}, \frac{m_{\ell^{\prime}}^{2}}{m_{A^{\prime}}^{2}}\right) \\
& \times\left[\frac{1}{2}+\frac{1}{2} \frac{m_{\ell}^{2}+6 m_{\ell} m_{\ell^{\prime}}+m_{\ell^{\prime}}^{2}}{m_{A^{\prime}}^{2}}-\frac{\left(m_{\ell}^{2}-m_{\ell^{\prime}}^{2}\right)^{2}}{m_{A^{\prime}}^{4}}\right]
\end{aligned}
$$

with $m_{A^{\prime}}$ being a mass of $A^{\prime}$, and $D=\mu_{\ell}$ or $\mu^{\prime}$ for the CLFC or the CLFV decays, respectively.

\section{Light boson production in beam dump experiments}

In electron beam dump experiments, a new light boson $X\left(=\phi, a, Z^{\prime}, A^{\prime}\right)$ is produced by the bremsstrahlung process with nucleons in target materials. When the light bosons have CLFV interactions with electron and muon, they also can be produced through flavor violating bremsstrahlung processes shown in figure 1. The bremsstrahlung production process can be evaluated by using the cross section of electron-photon scattering, $e^{-}+\gamma \rightarrow$ $\ell+X(\ell=e, \mu)$, in the Weizsäcker-Williams approximation [75-77]. In this section, we show the differential cross sections of the scalar and vector boson production process for the interactions given in the previous section. Then, we give formulae to calculate the number of events in electron beam dump experiments. 

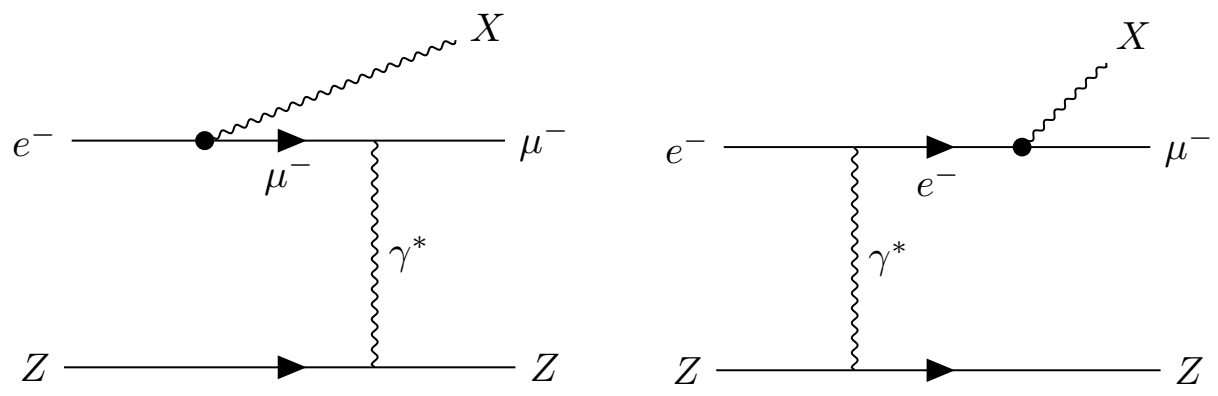

Figure 1. Light boson production through bremsstrahlung process with CLFV interaction by a target with the atomic number $Z$. The boson $X$ is either a (pseudo)scalar or vector.

\subsection{Differential cross section of bremsstrahlung process}

With the improved Weizsäcker-Williams approximation, the differential cross section of bremsstrahlung process of the light boson production by a target with atomic number $Z$, $e^{-}+Z \rightarrow \ell^{-}+Z+X\left(\ell=e, \mu, X=\phi, a, Z^{\prime}, A^{\prime}\right)$, is calculated by that of scattering one, $e^{-}+\gamma \rightarrow \ell^{-}+X$, as follows:

$$
\frac{d \sigma_{\text {brems }}}{d x}=\frac{\alpha \xi}{\pi} \frac{E_{0} x \beta_{X}}{1-x} \frac{d \sigma_{\text {scat }}}{d x}
$$

where $\alpha$ is the electromagnetic fine structure constant, $E_{0}$ is the energy of the injected electron beam, $\beta_{X}=\sqrt{1-m_{X}^{2} / E_{e}^{2}}$ is the kinematical factor, and $x=E_{X} / E_{e}$ with $E_{e(X)}$ being the energy of incident electron (produced light boson). The effective photon flux is denoted as $\xi$ [32], and the definition of $\xi$ is given in appendix A. The differential cross section of the scattering process with respect to $x$ is given by

$$
\begin{aligned}
\frac{d \sigma_{\text {scat }}}{d x}=\frac{\alpha g_{X}^{2}}{2 E_{0}} \frac{1-x}{x}[ & f_{1}(x) \frac{\tilde{U}_{1}^{\ell}}{m_{X}^{2}}+f_{2}(x) \frac{\tilde{U}_{2}^{\ell}}{E_{e}^{2} x} \\
& \left.\quad-f_{3}(x)\left(x \frac{m_{X}^{2} \tilde{U}_{3}^{\ell}}{\left(E_{e}^{2} x\right)^{2}}-\left(1-a_{1} x+r_{e} x^{2}\right) \frac{m_{X}^{4} \tilde{U}_{4}^{\ell}}{\left(E_{e}^{2} x\right)^{3}}\right)\right],
\end{aligned}
$$

where $g_{X}$ and $m_{X}$ are the coupling constant and mass of the light boson $X$, respectively. In the square bracket, $\tilde{U}_{n}^{\ell}(n=1-4)$ are functions of the maximal angle $\theta_{\text {max }}$ determined by the angular acceptance of the detector, and $\eta_{\ell}$ is given by

$$
\eta_{\ell}=\frac{m_{X}^{2}}{E_{e}^{2}} \frac{1-x}{x^{2}}+\frac{m_{e}^{2}}{E_{e}^{2}}+\frac{m_{\ell}^{2}-m_{e}^{2}}{E_{e}^{2} x},
$$

where $m_{\ell}$ is the mass of lepton $\ell$. The definition and approximate form of $\tilde{U}_{n}^{\ell}$ are given in appendix B. The functions $f_{1}(x), f_{2}(x)$, and $f_{3}(x)$ depend on the final state lepton $\ell$ and the types of the light boson $X$. For convenience, we define

$$
r_{\ell}=\frac{m_{\ell}^{2}}{m_{X}^{2}}
$$


and

$$
\begin{aligned}
& a_{1}=1+r_{e}-r_{\ell}, \\
& a_{2}=1-r_{e}-r_{\ell}, \\
& a_{3}=2+r_{e}+r_{\ell}-2 \sqrt{r_{e} r_{\ell}}, \\
& a_{4}=2-r_{e}-r_{e}^{2}-r_{\ell}-r_{\ell}^{2}+6 \sqrt{r_{e} r_{\ell}}+2 r_{e} r_{\ell} .
\end{aligned}
$$

Note that $\ell=e$ corresponds to the case of the CLFC interactions while $\ell=\mu$ to the CLFV interactions. Thus, the difference between the CLFC and CLFV interactions arises in the third term of $\eta_{\ell}$ as well as $r_{\ell}$ and $g_{X}$.

For the scalar interactions given in eq. (2.1), $X=\phi$ and $g_{\phi}=1$. The functions $f_{i}(x)$ in eq. (3.2) are

$$
f_{1}(x)=0, \quad f_{2}(x)=S_{1} \frac{x^{2}}{2}, \quad f_{3}(x)=\left(a_{2} S_{1}-4 \sqrt{r_{e} r_{\ell}} S_{2}\right)(1-x),
$$

where $S_{1}$ and $S_{2}$ are defined just below eq. (2.4). For the pseudoscalar interactions given in eq. (2.5), $X=a$ and $g_{a}^{2}=4\left|c_{e e}\right|^{2} m_{e}^{2} / \Lambda^{2}$ for the CLFC process, while $g_{a}^{2}=\left|c_{\mu e}\right|^{2}\left(m_{e}+\right.$ $\left.m_{\mu}\right)^{2} / \Lambda^{2}$ for the CLFV process. The functions $f_{i}(x)$ are given by

$$
f_{1}(x)=0, \quad f_{2}(x)=x^{2}, \quad f_{3}(x)=2\left(a_{2}+2 \sqrt{r_{e} r_{l}}\right)(1-x) .
$$

For the vector interactions given in eq. (2.8), $X=Z^{\prime}$ and $g_{Z^{\prime}}=g^{\prime} s^{2}$ for the CLFC process, while $g_{Z^{\prime}}=g^{\prime} s c$ for the CLFV process. The functions $f_{i}(x)$ are given by

$$
f_{1}(x)=0, \quad f_{2}(x)=4-4 x+a_{3} x^{2}, \quad f_{3}(x)=2 a_{4}(1-x) .
$$

For the dipole interactions given in eq. (2.12), $X=A^{\prime}$ and $g_{A^{\prime}}=\mu_{e} m_{A^{\prime}}$ for the CFLC process, while $g_{A^{\prime}}=\mu^{\prime} m_{A^{\prime}}$ for the CLFV process. The functions $f_{i}(x)$ are

$$
f_{1}(x)=4 x, \quad f_{2}(x)=x\left(x+2\left(r_{\ell}-r_{e}\right)(x-2)\right), \quad f_{3}(x)=2 a_{4}(1-x) .
$$

Figures 2, 3, 4, and 5 show the differential cross sections of the bremsstrahlung process for the scalar, pseudoscalar, vector, and dipole interactions with eqs. (3.6), (3.7), (3.8), and (3.9), respectively, as a function of the $X$ boson mass. In the left panels, $E_{e}$ is fixed to be $20 \mathrm{GeV}$, and $x$ is taken to be 1.0 (red), 0.9 (green), and 0.5 (blue). In the right panels, $E_{e}$ is varied as 20 (red), 10 (green), and 3 (blue) GeV, and $x$ is fixed to be 1 . Solid and dashed curves correspond to the CLFC and CLFV interactions, respectively.

In figure 2, the coupling constants are taken to be $y_{e}=y_{e \mu}=y_{\mu e}=10^{-6}$ as an illustrating example. In the left panel, the CLFV differential cross sections are much smaller than the CLFC ones with the same $x$. This is because $\tilde{U}_{n}^{\ell}$ are decreasing functions of $\eta_{\ell}$, and $\eta_{\mu}$ is larger than $\eta_{e}$. The CLFC differential cross sections increase by order of magnitudes as $x$ approaches unity because the second term in eq. (3.2) is much larger than the other terms. These with $x<1$ also rapidly decrease as $m_{\phi}$ becomes large since $\eta_{e}$ scales as $m_{\phi}^{2} / E_{e}^{2}$. On the other hand, the CLFV differential cross sections are rather constant because $\eta_{\mu}$ scales as $m_{\mu}^{2} / E_{e}^{2}$ for $m_{\phi}<m_{\mu}$. For $m_{\phi}$ larger than $m_{\mu}, r_{\mu} \ll 1$ and 

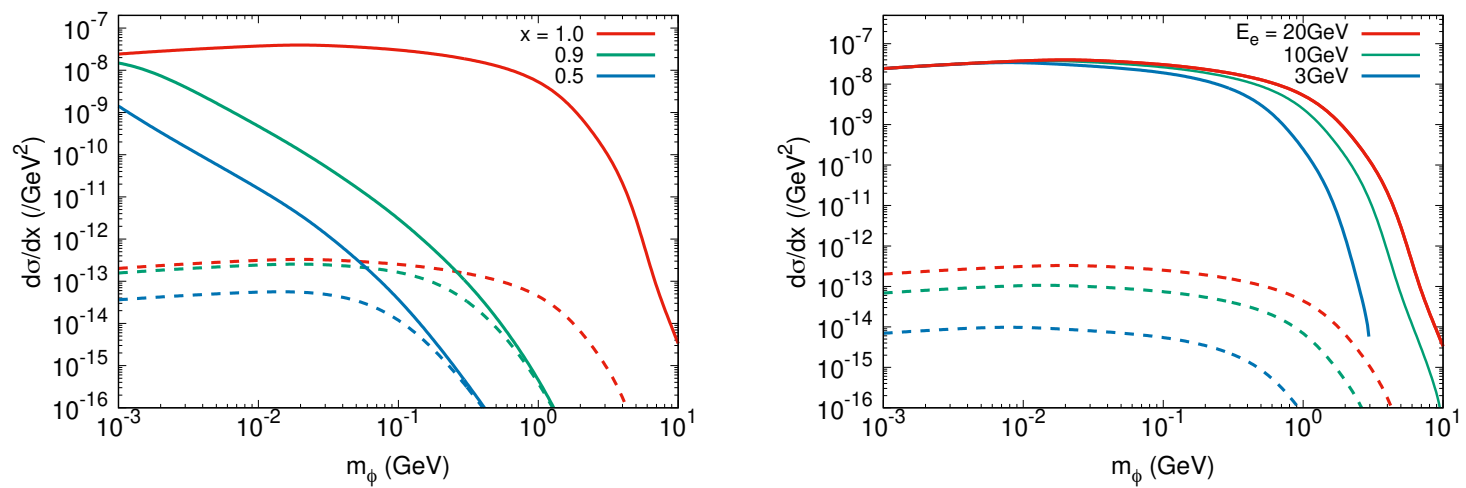

Figure 2. Differential cross section $d \sigma_{\text {brems }} / d x$ for the scalar interaction. Solid and dashed curves represent the CLFC and CLFV case, respectively, and the coupling constants are taken to be $y_{e}=y_{e \mu}=y_{\mu e}=10^{-6}$. Left panel: for $x=1.0$ (red), 0.9 (green), and 0.5 (blue), while fixing $E_{e}$ as $20 \mathrm{GeV}$. Right panel: for $E_{e}=20$ (red), 10 (green), and 3 (blue) GeV, while fixing $x$ as 1.0.
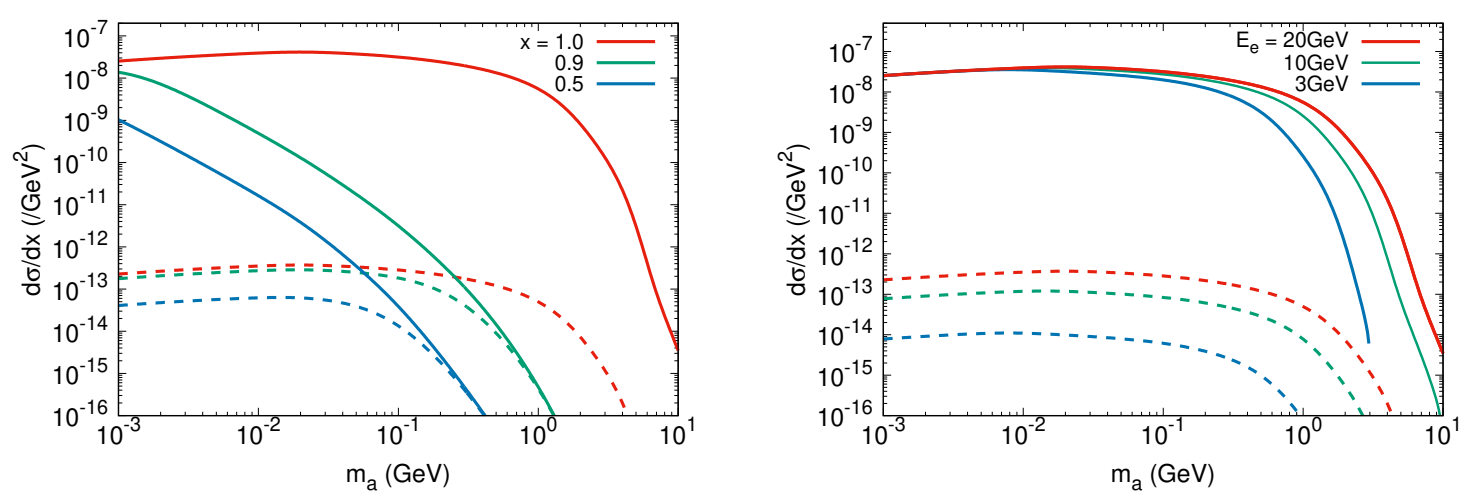

Figure 3. The same plots as figure 2 for the pseudoscalar interaction. The coupling constants are taken to be $c_{e e} / \Lambda=10^{-3} \mathrm{GeV}^{-1}$ and $c_{e \mu}=c_{\mu e}=10^{-2} c_{e e}$.

$\eta_{\mu} \simeq \eta_{e}$, therefore the CLFC and CLFV cross sections asymptotically approach to each other. In the right panel, the CLFC differential cross sections with $x=1$ are similar to different $E_{e}$ while the CLFV ones change order of magnitudes. For $x=1$, eq. (3.2) for the CLFC process is approximated as

$$
\frac{d \sigma_{\mathrm{brems}}}{d x} \simeq \frac{\alpha^{2}}{2 \pi} \xi \beta_{\phi} \frac{S_{1}}{4 m_{e}^{2}},
$$

where we have used $\tilde{U}_{2}^{e} \simeq 1 / 2 \eta_{e}=E_{e}^{2} / 2 m_{e}^{2}$. The effective photon flux is almost constant for $m_{\phi}<1 \mathrm{GeV}$. Thus, the electron energy dependence only comes from $\beta_{\phi}$ which is almost unity unless close to the threshold. In the CLFV process, on the other hand, the electron energy dependence remains in $\tilde{U}_{2}^{e} / E_{e}^{2}$. From both panels, it can be understood that the main contributions to the signal event come from the CLFC process with $0.9 \lesssim x \lesssim 1$.

Figure 3 shows the same plots as figure 2 for the pseudoscalar interactions. The coupling constants are fixed to $c_{e e} / \Lambda=10^{-3} \mathrm{GeV}^{-1}$ and $c_{e \mu}=c_{\mu e}=10^{-2} c_{e e}$ as an illustrating example. It can be seen that the shape of the differential cross sections is almost identical 

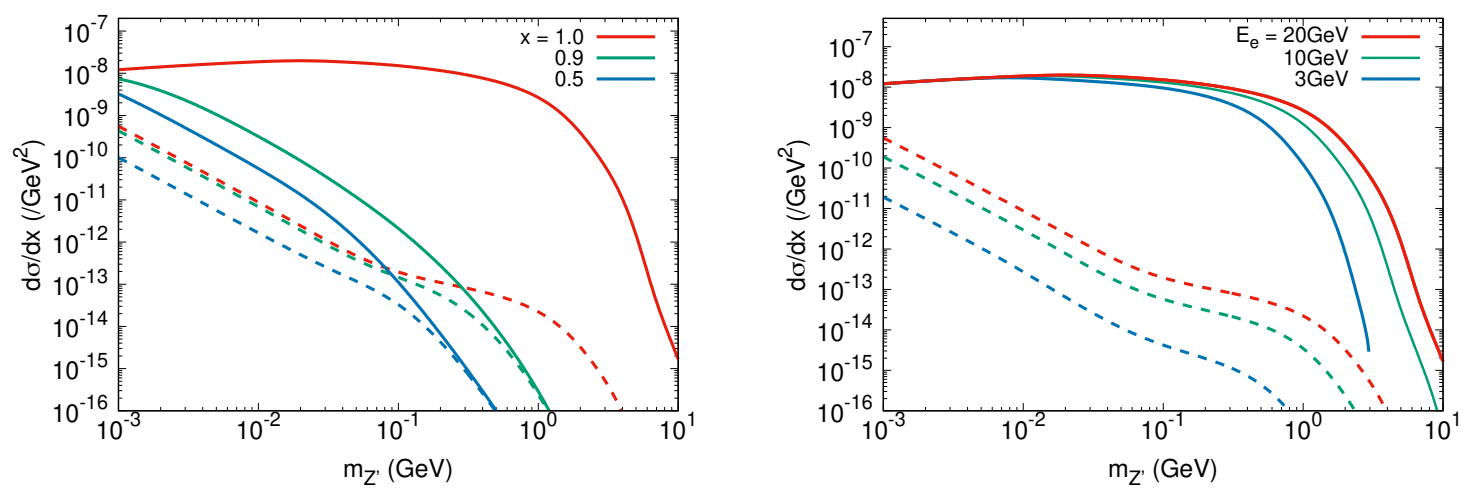

Figure 4. The same plots as figure 2 for the vector interaction. The coupling constants are taken to be $g^{\prime}=10^{-6}$ and $\theta=\pi / 4$.
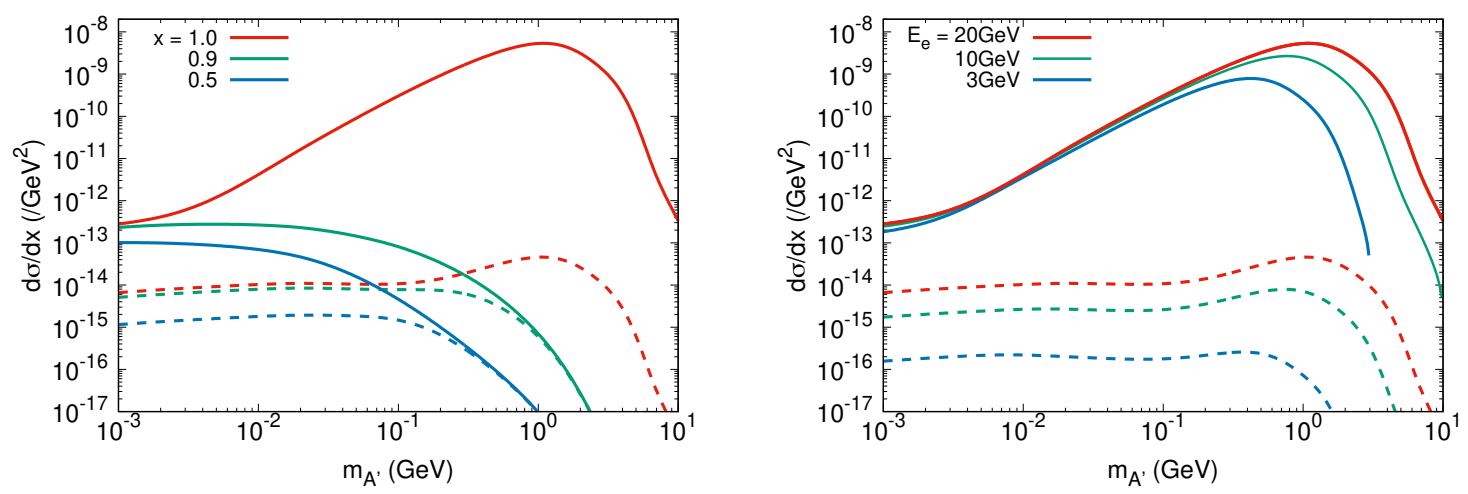

Figure 5. The same plot as figure 2 for the dipole interaction. The coupling constants are taken to be $\mu_{e}=\mu^{\prime}=10^{-6} \mathrm{GeV}^{-1}$.

to those for the scalar interaction case. Comparing the functions $f_{i}(x)$ in eq. (3.7) for the pseudoscalar case with those in eq. (3.6) for the scalar case, the difference is only $\sqrt{r_{e} r_{\mu}}$ in $f_{3}(x)$, which is smaller than $a_{2}$. Thus, the behavior of the differential cross sections for the pseudoscalar interactions is similar to that for the scalar interactions.

Figure 4 shows the same plots as figure 2 for the vector interactions. The coupling constant and the mixing angle are fixed to $g^{\prime}=10^{-6}$ and $\theta=\pi / 4$, respectively. Contrary to the scalar interaction cases, the CLFV differential cross sections decrease as $m_{Z^{\prime}}$ increases. This is because $f_{2}(x)$ in eq. (3.8) is dominated by $r_{\mu} x^{2}$ for small $m_{Z^{\prime}}$. The behavior of the CLFC differential cross sections can be understood in the same way as in the scalar interaction case. Figure 5 shows the same plots for the dipole interactions. The dipole moments are fixed to $\mu_{e}=\mu^{\prime}=10^{-6} \mathrm{GeV}^{-1}$. In this case, the differential cross sections are proportional to $m_{A^{\prime}}^{2}$. These increase as $m_{A^{\prime}}$ increases, and then go to zero as the mass reaches the kinematical threshold. From figures 4 and 5, it can be seen that the dominant contributions to the signal events come from the CLFC differential cross sections with $0.9 \lesssim x \lesssim 1$, also for the vector and dipole interactions. 


\subsection{Number of signal events}

The number of the signal events can be calculated by the following formula [32],

$$
\begin{aligned}
N=N_{e} & \frac{N_{\mathrm{avo}} X_{0}}{A} \sum_{\ell=e, \mu} \int_{m_{X}}^{E_{0}-m_{\ell}} d E_{X} \int_{E_{X}+m_{\ell}}^{E_{0}} d E_{e} \int_{0}^{T_{\mathrm{sh}}} d t \\
& \times\left[\left.I_{e}\left(E_{0}, E_{e}, t\right) \frac{1}{E_{e}} \frac{d \sigma_{\mathrm{brems}}}{d x}\right|_{x=\frac{E_{X}}{E_{e}}} e^{-L_{\mathrm{sh}} / L_{X}}\left(1-e^{-L_{\mathrm{dec}} / L_{X}}\right)\right] \operatorname{Br}\left(X \rightarrow e^{+} e^{-}\right),
\end{aligned}
$$

where $N_{e}$ is the number of electrons in the injected beam, $N_{\text {avo }} \simeq 6 \times 10^{23} \mathrm{~mol}^{-1}$ the Avogadro's number, $X_{0}$ the radiation length of the target, $A$ the target atomic mass in $\mathrm{g} / \mathrm{mol}$, and $T_{\mathrm{sh}} \equiv \rho_{\mathrm{sh}} L_{\mathrm{sh}} / X_{0}$ with $\rho_{\mathrm{sh}}$ being the density of the shield. The lengths of shield and decay region are denoted as $L_{\mathrm{sh}}$ and $L_{\mathrm{dec}}$, respectively. The decay length in laboratory frame and branching ratio of $X$ are denoted by $L_{X}$ and $\operatorname{Br}\left(X \rightarrow e^{+} e^{-}\right)$, respectively. The energy distribution of electrons after passing through $t$ radiation lengths in the beam dump is denoted by $I_{e}$ and given by [78]

$$
I_{e}\left(E_{0}, E_{e}, t\right)=\frac{1}{E_{0}} \frac{\left[\ln \left(E_{0} / E_{e}\right)\right]^{b t-1}}{\Gamma(b t)},
$$

where $b=4 / 3$. This energy distribution function sharply peaks around $t \simeq 0$ for $E_{e}=E_{0}$, and the integration over $t$ can diverge. To avoid such a divergence, we split the interval of $t$ into two parts by a cut $t_{\text {cut }}$, following ref. [79].

\section{Electron beam dump constraints}

In this section, we show the constraints from the electron beam dump experiment, E137 [28], for the scalar, pseudoscalar, vector, and dipole interactions with CLFV. In the E137 experiment, the electron beam with energy $20 \mathrm{GeV}$ is injected into an aluminium target. The total number of electrons dumped into the target is $1.86 \times 10^{20}$, which corresponds to $30 \mathrm{C}$. The detector consists of an electromagnetic shower counter with dimensions $2 \mathrm{~m} \times$ $3 \mathrm{~m}$ in the first phase, and $3 \mathrm{~m} \times 3 \mathrm{~m}$ in the second phase. It has a $204 \mathrm{~m}$ decay region placed at $179 \mathrm{~m}$ downstream to the dump, ${ }^{2}$ and a hill placed between the target and decay region plays a role of the shield. We summarize the setup of the E137 experiment in table 1. In order to take into account the geometrical acceptance, we set $\theta_{\max }$ in eq. (B.1) as $\theta_{\max }=1.5 /(179+204)$. The E137 collaboration reported null results for the energy above $3 \mathrm{GeV}$ in the search for axion-like particles, so that we regard the regions predicting $N>3$ events as the $95 \%$ C.L. exclusion regions and set the lower energy threshold of $3 \mathrm{GeV}$ on the integral over $E_{X}$ in eq. (3.11).

\footnotetext{
${ }^{2}$ One may consider the produced muon could penetrate the shield and be observed by the detector in beam dump experiments. Using the continuous-slowing-down-approximation range [80], the range for the muon to be stopped is estimated at $\sim 50 \mathrm{~m}$ at most for the E137 experiment. The range is $1 / 3$ times shorter than the shield length. Therefore, the produced muon would be stopped in the shield.
} 


\begin{tabular}{|cccccc|}
\hline target & $\begin{array}{c}\text { radiation length } \\
X_{0}\left[\mathrm{~g} / \mathrm{cm}^{2}\right]\end{array}$ & $\begin{array}{c}\text { beam energy } \\
E_{0}[\mathrm{GeV}]\end{array}$ & $\begin{array}{c}\text { total electrons } \\
N_{e}\end{array}$ & $\begin{array}{c}\text { shield length } \\
L_{\mathrm{sh}}[\mathrm{m}]\end{array}$ & $\begin{array}{c}\text { decay volume length } \\
L_{\text {dec }}[\mathrm{m}]\end{array}$ \\
\hline${ }_{13}^{26.98} \mathrm{Al}$ & 24.01 & 20 & $1.86 \times 10^{20}$ & 179 & 204 \\
\hline
\end{tabular}

Table 1. Setup of the E137 experiment.

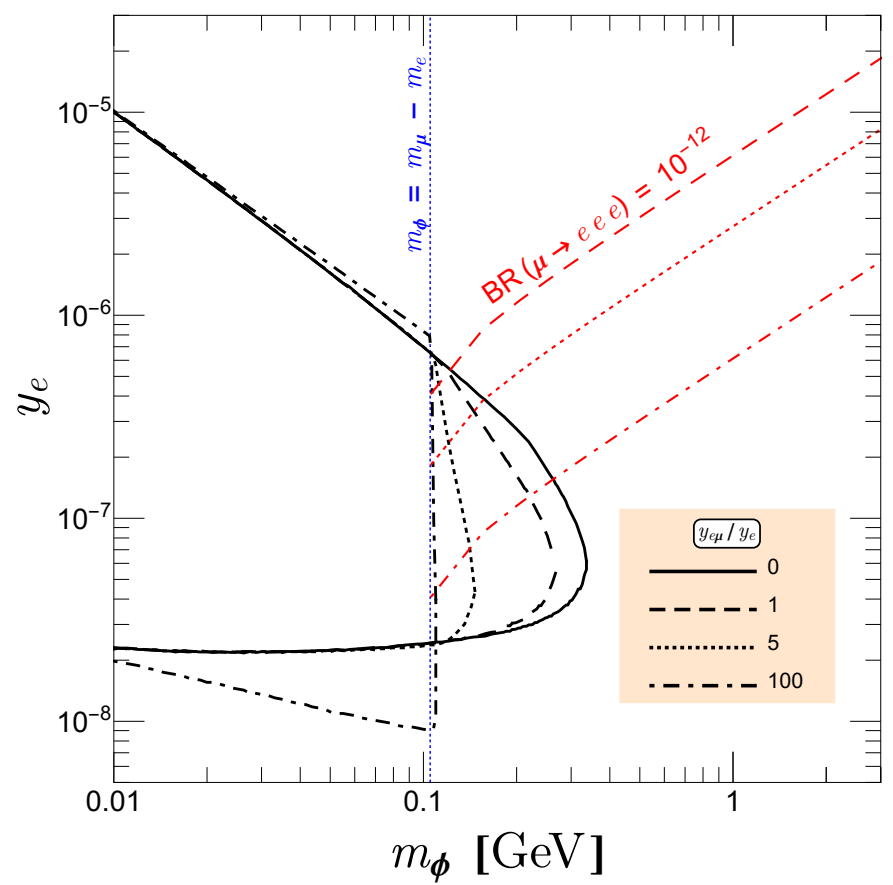

Figure 6. Exclusion region from the E137 experiment for the scalar interaction. The CLFV coupling is taken to $y_{e \mu} / y_{e}=0$ (solid), 1 (dashed), 5 (dotted), and 100 (dot-dashed). Red curves represent the constraints from $\mu \rightarrow$ eee, and blue vertical line corresponds to $m_{\phi}=m_{\mu}-m_{e}$.

Figure 6 shows the exclusion regions in $m_{\phi}-y_{e}$ plane at 95\% C.L. from E137 for the scalar interaction case. For simplicity, we assume $y_{e}=y_{\mu}=y_{\tau}, y_{e \mu}=y_{\mu e}$ and $y_{e}, y_{e \mu}$ are real. Solid, dashed, dotted, and dot-dashed curves correspond to $y_{e \mu} / y_{e}=0,1,5$, and 100, respectively. Among them, $y_{e \mu} / y_{e}=0$ (the solid curve) corresponds to the CLFC case. For the CLFC case, we have checked that our result is in good agreement with the result derived in ref. [81], except that we here include the interaction with muons as well as electrons. For the cases of nonzero $y_{e \mu}$, there are strong constraints from CLFV decays of muons. The regions above the red curves are excluded by $\mu \rightarrow$ eee, while most regions of the left side of the blue vertical dotted line are excluded by $\mu \rightarrow e \phi$. The upper bounds of these decay branching ratios are listed in table 2, and the partial widths of these CLFV decays are given in appendix C. Note that constraints from $\mu \rightarrow e \gamma$ are weaker than those from $\mu \rightarrow$ eee since the decay width of $\mu \rightarrow e \gamma$ is suppressed by the electromagnetic coupling and a loop factor in comparison with that of $\mu \rightarrow e e e$. As can be seen from the figure, most of the parameter regions are already excluded by these constraints. Nevertheless, we find that our $95 \%$ C.L. limit further excludes the parameter regions unconstrained by $\mu \rightarrow$ eee and $\mu \rightarrow e \phi$. The exclusion region shifts to lighter $m_{\phi}$ as $y_{e \mu} / y_{e}$ increases. As we explained 


\begin{tabular}{|c|c|c|c|c|}
\hline $\operatorname{Br}(\mu \rightarrow e e e)$ & $\operatorname{Br}(\mu \rightarrow e \gamma)$ & \multicolumn{3}{|c|}{$\operatorname{Br}(\mu \rightarrow e X)$} \\
\hline$m_{X}>m_{\mu}-m_{e}$ & $m_{X}>m_{\mu}-m_{e}$ & $13<m_{X}<80$ & $47.8<m_{X}<95.1$ & $98.1<m_{X}<103.5$ \\
\hline$<1.0 \times 10^{-12}[82]$ & $<4.2 \times 10^{-13}[72]$ & $\lesssim 10^{-5}[83]$ & $\lesssim 10^{-5}[84]$ & $\lesssim 2 \times 10^{-4}[85]$ \\
\hline
\end{tabular}

Table 2. Upper bounds on the branching ratios of $\mu \rightarrow e e e, \mu \rightarrow e \gamma$, and $\mu \rightarrow e X$. Masses are in $\mathrm{MeV}$.

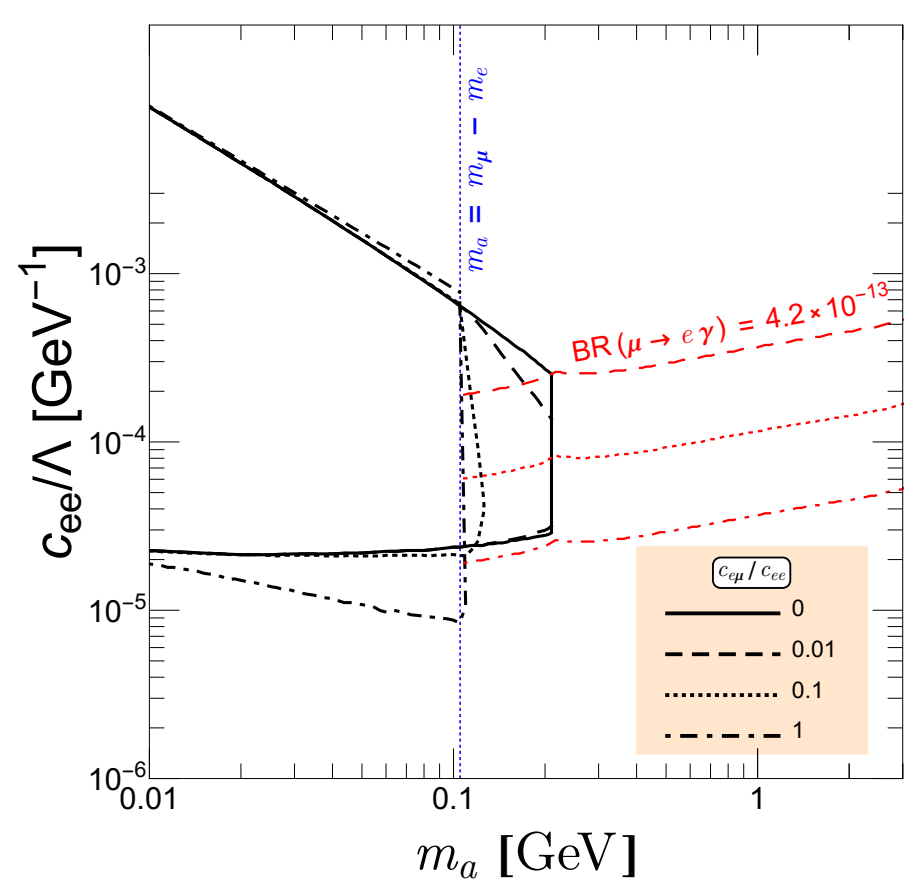

Figure 7. The same plots as figure 6 for the pseudoscalar interaction. The CLFV coupling is taken to $c_{e \mu} / c_{e e}=0$ (solid), 0.01 (dashed), 0.1 (dotted), and 1 (dot-dashed).

in section 3, the differential cross sections are mainly determined by the CLFC ones for $y_{e \mu} / y_{e}<10$. Therefore, in this case, the effects of the flavor violating couplings appear only in the total decay width. Once the threshold of $\phi \rightarrow e \mu$ opens, the decay length and branching ratio of $\phi \rightarrow e e$ become smaller. Then, the expected number of the signal events reduces, resulting in the exclusion regions shown in figure 6 . For $y_{e \mu} / y_{e}=100$, the CLFV differential cross sections are comparable to the CLFC ones. Then, through the CLFV production process, the expected number of the scalar boson produced in the beam dump is increased, making the exclusion regions wider especially in the small $y_{e}$ regions. Such a large $y_{e \mu}$, however, makes the decay length and the branching ratio so small that no signal events are expected above the threshold of $\mu \rightarrow e \phi$.

Figure 7 shows the exclusion plot in $m_{a}-c_{e e} / \Lambda$ plane for the pseudoscalar interaction. For the plot of this figure, we assume $c_{e e}=c_{\mu \mu}=c_{\tau \tau}, c_{e \mu}=c_{\mu e}$, and $\Lambda=10^{3} \mathrm{GeV}$. The ratio of the CLFV coupling to the CLFC one is varied as $c_{e \mu} / c_{e e}=0$ (solid), 0.01 (dashed), 0.1 (dotted), and 1 (dot-dashed). The red curves and the blue line represent the constraints from $\mu \rightarrow e \gamma$ and $\mu \rightarrow e a$, respectively. For the pseudoscalar interaction case, the constraint from $\mu \rightarrow e \gamma$ is stronger than that from $\mu \rightarrow 3 e$ because of the enhancement 
of heavy lepton masses propagating in the loop. ${ }^{3}$ As with refs. $[37,86]$, we find that the exclusion region for the CLFC process disappears in $m_{a}>2 m_{\mu}$. This is because $a \rightarrow \mu \mu$ opens, and the lifetime of the pseudoscalar boson becomes so short that it cannot reach the detector. One can find similar behavior of the exclusion plot for the CLFV process. For the cases of $c_{e \mu} / c_{e e} \geq 0.1$, the exclusion regions disappear in $m_{a}>m_{\mu}+m_{e}$, since $a \rightarrow e \mu$ opens and the lifetime of the pseudoscalar boson becomes very short. From figure 7 , one can see that our results exclude the unconstrained parameter regions for the ratio smaller than 1 . Here, it should be noted that the pseudoscalar boson can also be produced by the pair-annihilation and the Primakoff production; inclusion of these production processes would make the exclusion region somewhat wider [41].

Figure 8 shows the exclusion plot in $m_{Z^{\prime}} g^{\prime}$ plane for the vector interaction. The mixing angle is varied as $\theta=0.2$ (solid), 0.5 (dashed), 1 (dotted), and $\pi / 2$ (dot-dashed). The red curves and the blue line represent the same constraints as in figure 6 . In contrast to the cases of the scalar and pseudoscalar interactions, the exclusion regions become wider as the effect of CLFV becomes large. This is because a nonzero $\theta$ induces interactions between $Z^{\prime}$ and electrons, ${ }^{4}$ increasing the production cross sections and the branching ratio of $Z^{\prime} \rightarrow e e$ simultaneously. For $\theta>0.5$, the constraint from E137 experiment excludes the parameter regions for $m_{Z^{\prime}}>m_{\mu}-m_{e}$, which are not constrained by $\mu \rightarrow e e e$ and $\mu \rightarrow e Z^{\prime}$. When $\theta=\pi / 2$, the interaction with muon vanishes, and the exclusion region coincides with that for the minimal $\mathrm{U}(1)_{L_{e}-L_{\tau}}$ model. Here, we note that our result for the minimal $\mathrm{U}(1)_{L_{e}-L_{\tau}}$ model is somewhat larger than the result derived in ref. [34], probably due to the lack of efficiency factors of the experiment in our calculations. In order to confirm the validity of our calculation, we have also checked the consistency with the result obtained in ref. [87].

Figure 9 shows the exclusion plot in $m_{A^{\prime}}-\mu_{e}$ plane for the dipole interactions with the assumption of $\mu_{e}=\mu_{\mu}=\mu_{\tau}$ for simplicity. The CLFV dipole coupling are taken to $\mu^{\prime} / \mu_{e}=0$ (solid), 1 (dashed), 10 (dotted), and 100 (dot-dashed). In this case, the constraint from the E137 experiment also excludes the parameter region below the $\mu \rightarrow$ eee limit. The behavior of the exclusion region is similar to the case of the scalar interactions.

\section{$5 \quad$ Flavor violating decay signal}

In the previous section, we have only considered the CLFC decay of $X \rightarrow e e$ as the signal of the vector and scalar boson, based on the setup and analyses of the E137 experiment. Under the presence of the CLFV interactions, the light bosons can decay into $e \bar{\mu}$ and $\bar{e} \mu$ above their kinematical threshold. These decay modes are smoking gun signatures of CLFV interactions in the dark sector. Searches for these decays will bring further information on the CLFV couplings.

To illustrate the impact of the CLFV decay searches, we have demonstrated analyses for the scalar CLFV decays with the setup of the E137 experiment in table 1. Figure 10

\footnotetext{
${ }^{3}$ The CLFV decay of the muon to the electron and photon, $\mu \rightarrow e \gamma$, are induced by diagrams at one-loop level. For the pseudoscalar case, the Barr-Zee type diagrams at the two-loop level, however, give significant contributions to the branching ratio of $\mu \rightarrow e \gamma$. See ref. [52] for instance.

${ }^{4}$ Even in the case of $\theta=0$, interactions between $Z^{\prime}$ and electrons arise at one-loop level, which yields narrow exclusion regions around $m_{Z^{\prime}}=0.001-0.003 \mathrm{GeV}[34]$.
} 


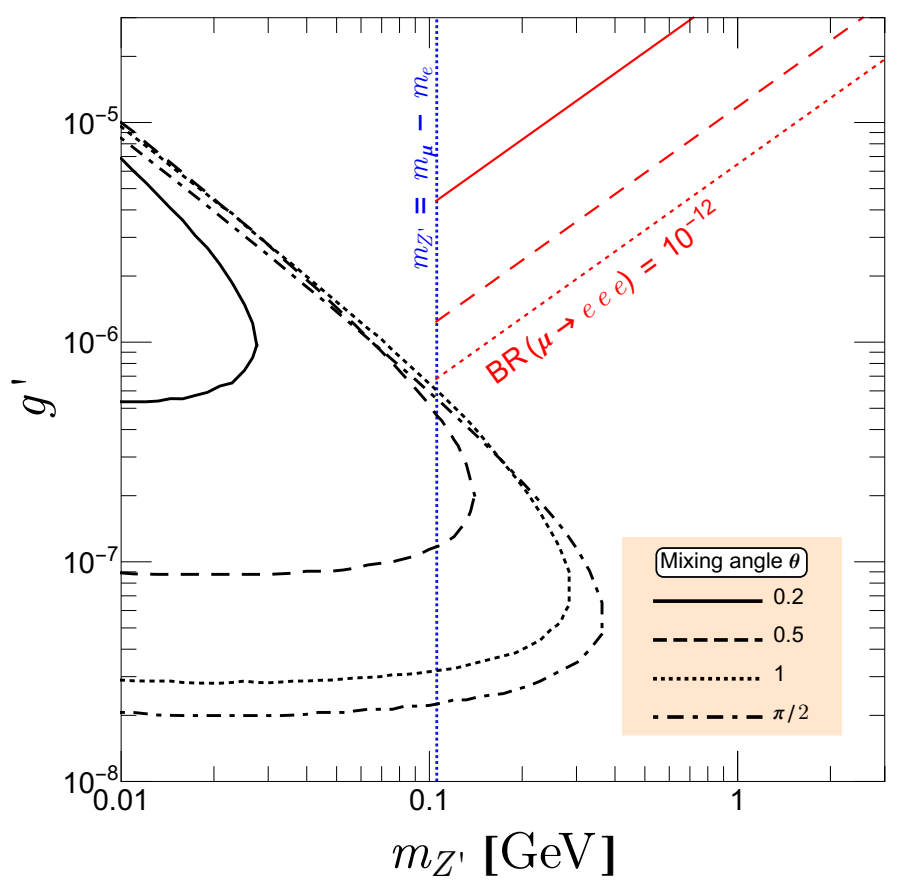

Figure 8. The same plots as figure 6 for the vector interaction. The mixing angle is taken to 0.2 (solid), 0.5 (dashed), 1 (dotted), and $\pi / 2$ (dot-dashed).

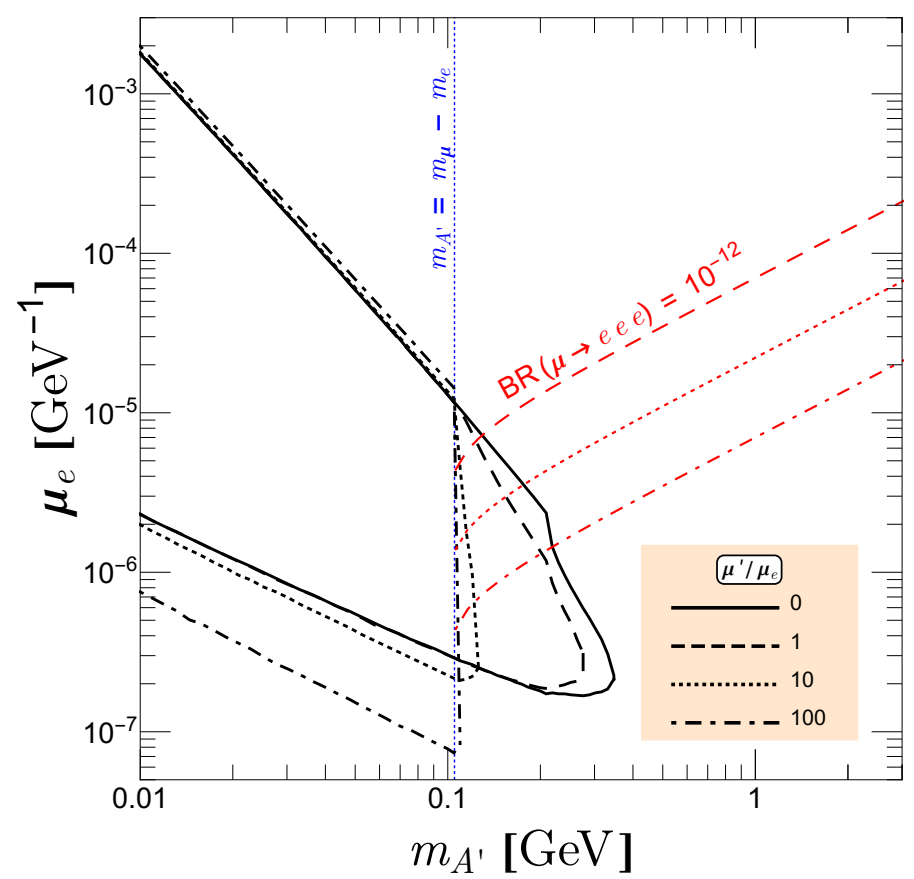

Figure 9. The same plots as figure 6 for the dipole interaction. The dipoles are taken to $\mu^{\prime} / \mu_{e}=0$ (solid), 1 (dashed), 10 (dotted), and 100 (dot-dashed). 


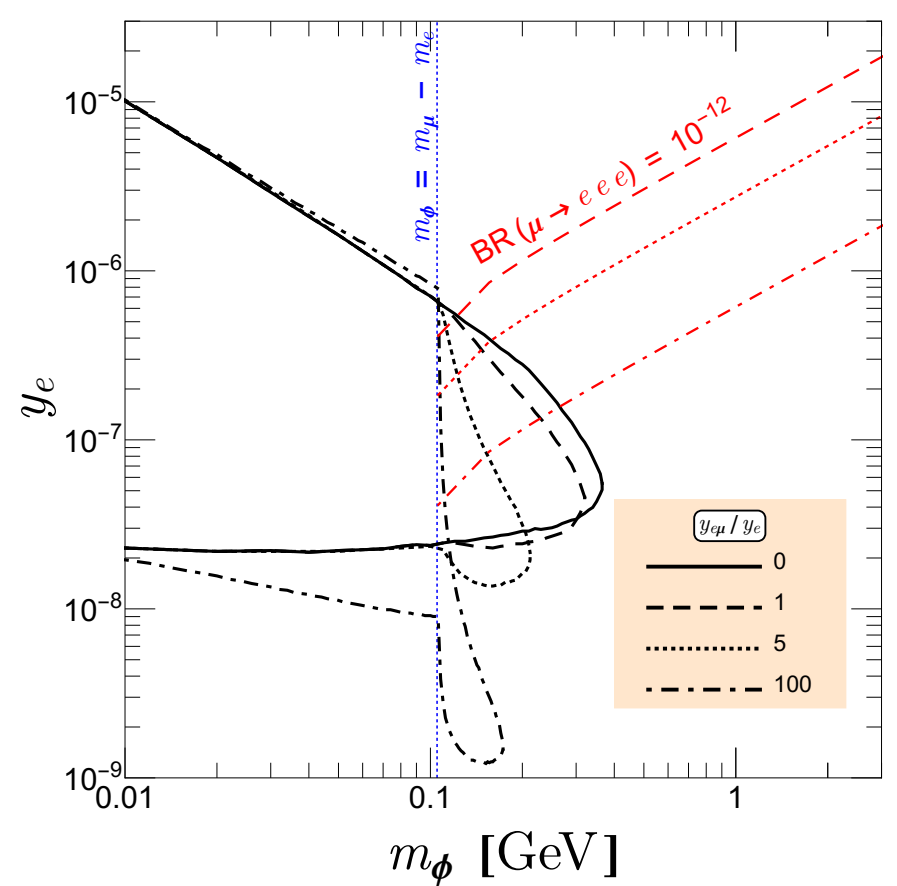

Figure 10. Presumed exclusion region to $\phi \rightarrow e e, e \mu, \mu \mu$ decays in the E137 experiment for the scalar interaction. The parameters are taken as $y_{e \mu} / y_{e}=0$ (solid), 1 (dashed), 5 (dotted), and 100 (dot-dashed).

shows presumed exclusion regions with 95\% C.L. for $\phi \rightarrow e e, e \mu, \mu \mu$. The solid, dashed, dotted, and dot-dashed curves correspond to $y_{e \mu} / y_{e}=0,1,5$, and 100, respectively. Compared with figure 6 , smaller coupling and larger mass regions can be excluded above the $e \mu$ threshold. This is because, for $m_{\phi} \geq m_{e}+m_{\mu}\left(2 m_{\mu}\right)$, the decay branch of $\phi \rightarrow e \mu(\mu \mu)$ opens, and the decay length of the light boson shortens. The smaller coupling is, therefore, preferred to reach the detector of the E137 experiment. Since these regions cannot be excluded only by analyzing the ee decay search, the CLFV couplings can be further constrained in these regions. Thus, it is important to search for not only the CLFC decays but also the CLFV decays as signals in future beam dump experiments. FASER (ForwArd Search ExpeRiment) [88-90], which will start next year, will be able to perform the searches for the dark photon decay into $e \mu$ pairs. Theoretical analyses will be shown in ref. [91].

\section{Summary}

We have studied the constraints from the electron beam dump experiment taking into account the charged lepton flavor violating interactions. The Yukawa, Axion-like particles, vector, and dipole type interactions are considered, and the excluded regions have been derived by the search for $X \rightarrow e e$ in the E137 experiment. We have found that the parameter regions unconstrained by $\mu \rightarrow e e e$ or $\mu \rightarrow e \gamma$ as well as $\mu \rightarrow e X$ can be excluded for four types of the interactions. The exclusion regions depend on the CLFV and CLFC couplings as well as the boson mass. New bounds can be derived when the ratios of the 
CLFV coupling to the CLFC one are $\lesssim 100$ for the scalar and dipole interactions, and $\lesssim 1$ for the pseudoscalar interaction case. That for the vector interaction is also obtained for the CLFV mixing angle $\gtrsim 0.5$. For the illustrative purpose, we have also derived the presumed exclusion region for the search of $\phi \rightarrow e e, e \mu, \mu \mu$ decays in the same setup with the E137 experiment. It has been found that the exclusion regions can be extended and the CLFV couplings can be further constrained in those regions. Such searches and analyses for the CLFV decays will be important to new physics searches in future experiments.

\section{Acknowledgments}

This work was supported by JSPS KAKENHI Grant Number JP18H01210 [TA, TS], JP18K03651 [TS], and MEXT KAKENHI Grant Number JP18H05543 [TS].

\section{A Effective photon flux}

The effective photon flux $\xi$ produced by an atom with the mass number $A$ and atomic number $Z$ is defined by [32]

$$
\xi=\int_{t_{\min }}^{t_{\max }} d t \frac{t-t_{\min }}{t^{2}} G_{2}(t),
$$

where $t_{\min }=\left(m_{X}^{2} / 2 E_{e}\right)^{2}$ and $t_{\max }=m_{X}^{2}$. The electric form factor $G_{2}(t)$ is given by elastic and inelastic components as follows:

$$
G_{2}(t)=G_{2, \mathrm{el}}+G_{2, \text { inel }}
$$

The elastic electric form factor is given by

$$
G_{2, \mathrm{el}}=\left(\frac{a^{2} t}{1+a^{2} t}\right)^{2}\left(\frac{1}{1+\frac{t}{d}}\right)^{2} Z^{2},
$$

where $a=111 Z^{-1 / 3} / m_{e}$ and $d=0.164 \mathrm{GeV}^{2} A^{-2 / 3}$. The inelastic electric form factor is given by

$$
G_{2, \text { in }}=\left(\frac{a^{\prime 2} t}{1+{a^{\prime}}^{2} t}\right)^{2}\left(\frac{1+\frac{t}{4 m_{p}^{2}}\left(\mu_{p}^{2}-1\right)}{\left(1+\frac{t}{0.71 \mathrm{GeV}^{2}}\right)^{4}}\right)^{2} Z,
$$

where $a^{\prime}=773 Z^{-2 / 3} / m_{e}, \mu_{p}=2.79$, and $m_{p}$ is the proton mass.

The electric form factor is dominated by the elastic one in our study, and thus it scales by $Z^{2}$. See ref. [32] for more detail.

\section{B Function $\tilde{U}_{n}^{\ell}$}

The functions $\tilde{U}_{n}^{\ell}(n=1-4)$ are defined by

$$
\tilde{U}_{n}^{\ell}=\int_{0}^{\theta_{\max }} \frac{\sin \theta}{\left[\theta^{2}+\eta_{\ell}(x)\right]^{n}} d \theta
$$


where $\theta_{\max }$ is the maximal angle determined by angular acceptance of the detector, and $\eta_{\ell}(x)$ is given in eq. (3.3). For the small angle $\theta_{\max }, \tilde{U}_{n}^{\ell}$ can be approximated by the following formulae:

$$
\begin{aligned}
\tilde{U}_{1}^{\ell} \simeq & \frac{1}{2} \log \left(\frac{\eta_{\ell}+\theta_{\max }^{2}}{\eta_{\ell}}\right)+\frac{1}{12}\left(\eta_{\ell} \log \left(\frac{\eta_{\ell}+\theta_{\max }^{2}}{\eta_{\ell}}\right)-\theta_{\max }^{2}\right) \\
& +\frac{1}{480}\left(2 \eta_{\ell}^{2} \log \left(\frac{\eta_{\ell}+\theta_{\max }^{2}}{\eta_{\ell}}\right)-2 \eta_{\ell} \theta_{\max }^{2}+\theta_{\max }^{4}\right) \\
\tilde{U}_{2}^{\ell} \simeq & \frac{\theta_{\max }^{2}}{2 \eta_{\ell}\left(\eta_{\ell}+\theta_{\max }^{2}\right)}+\frac{1}{12}\left(-\frac{\eta_{\ell}}{\eta_{\ell}+\theta_{\max }^{2}}-\log \left(\frac{\eta_{\ell}+\theta_{\max }^{2}}{\eta_{\ell}}\right)+1\right) \\
& +\frac{1}{120}\left(\frac{\left(2 \eta_{\ell}+\theta_{\max }^{2}\right) \theta_{\max }^{2}}{2\left(\eta_{\ell}+\theta_{\max }^{2}\right)}-\eta_{\ell} \log \left(\frac{\eta_{\ell}+\theta_{\max }^{2}}{\eta_{\ell}}\right)\right) \\
\tilde{U}_{3}^{\ell} \simeq & \frac{\left(2 \eta_{\ell}+\theta_{\max }^{2}\right) \theta_{\max }^{2}}{4 \eta_{\ell}^{2}\left(\eta_{\ell}+\theta_{\max }^{2}\right)^{2}}-\frac{\theta_{\max }^{4}}{24 \eta_{\ell}\left(\eta_{\ell}+\theta_{\max }^{2}\right)^{2}} \\
& +\frac{1}{120}\left(\frac{1}{2} \log \left(\frac{\eta_{\ell}+\theta_{\max }^{2}}{\eta_{\ell}}\right)-\frac{\left(2 \eta_{\ell}+3 \theta_{\max }^{2}\right) \theta_{\max }^{2}}{4\left(\eta_{\ell}+\theta_{\max }^{2}\right)^{2}}\right) \\
\tilde{U}_{4}^{\ell} \simeq & \frac{\left(3 \eta_{\ell}^{2}+3 \eta_{\ell} \theta_{\max }^{2}+\theta_{\max }^{4}\right) \theta_{\max }^{2}}{6 \eta_{\ell}^{3}\left(\eta_{\ell}+\theta_{\max }^{2}\right)^{3}}-\frac{\left(3 \eta_{\ell}+\theta_{\max }^{2}\right) \theta_{\max }^{4}}{72 \eta_{\ell}^{2}\left(\eta_{\ell}+\theta_{\max }^{2}\right)^{3}}+\frac{\theta_{\max }^{6}}{720 \eta_{\ell}\left(\eta_{\ell}+\theta_{\max }^{2}\right)^{3}} .
\end{aligned}
$$

\section{Widths of CLFV decays of muons}

We here discuss the experimental limits on the CLFV decays of muons. The CLFV is tightly constrained by the rare muon decays, i.e., $\mu \rightarrow e \gamma$ and $\mu \rightarrow e e e$. The current strongest limits on the branching ratio of $\mu \rightarrow e \gamma$ is given by MEG [72] as $\operatorname{BR}(\mu \rightarrow e \gamma)<$ $4.2 \times 10^{-13}$. On the other hand, the strongest limits of $\mu \rightarrow e e e$ is given by SINDRUM [82] as $\operatorname{BR}(\mu \rightarrow e e e)<10^{-12}$. Though the constraint on $\operatorname{BR}(\mu \rightarrow e \gamma)$ is stronger than that on $\operatorname{BR}(\mu \rightarrow e e e)$, the decay width of $\mu \rightarrow e \gamma$ is suppressed by the electromagnetic coupling and a loop factor in comparison with that of $\mu \rightarrow e e e$. As a result, usually, the bound on CLFV couplings from $\mu \rightarrow$ eee is stronger than that from $\mu \rightarrow e \gamma$. We, therefore, take into account the constraint from $\mu \rightarrow$ eee for $m_{X}>m_{\mu}-m_{e}$, except for the case of the pseudoscalar interactions. For $m_{X}<m_{\mu}-m_{e}$, muons can decay into the light bosons. ${ }^{5}$ The TWIST collaboration [83] gives the limits on the branching ratio of $\mu \rightarrow e X$ as $\operatorname{BR}(\mu \rightarrow e X) \lesssim 10^{-5}$ for $m_{X}=13-80 \mathrm{MeV}$. The PIENU collaboration [84] also gives the upper limit as $\operatorname{BR}(\mu \rightarrow e X) \lesssim 10^{-5}-10^{-4}$ for $m_{X}=47.8-95.1 \mathrm{MeV}$. Moreover, ref. [85] gives the upper limit as $\operatorname{BR}(\mu \rightarrow e X) \lesssim 2 \times 10^{-4}$ for $m_{X}=98.1-103.5 \mathrm{MeV}$. These limits give much more severe constraints on the CLFV coupling than that from the electron beam dump, and then we focus on the region where the light boson is heavier than $m_{\mu}-m_{e}$.

The partial decay width of $\ell_{\alpha}^{-} \rightarrow \ell_{\beta}^{-} \ell_{\beta}^{-} \ell_{\beta}^{+}$, which is shown in figure 11 , is given by

$$
\Gamma=\frac{1}{\left(8 \pi m_{\alpha}\right)^{3}} \int_{s_{12}^{\min }}^{s_{12}^{\max }} d s_{12} \int_{s_{23}^{\min }}^{s_{23}^{\max }} d s_{23} \frac{1}{2} \sum_{\text {spin }}|M|^{2},
$$

\footnotetext{
${ }^{5}$ For axion-like-particles $a$, detailed study on the constraints of $\mu \rightarrow e a$ can be found in ref. [68].
} 

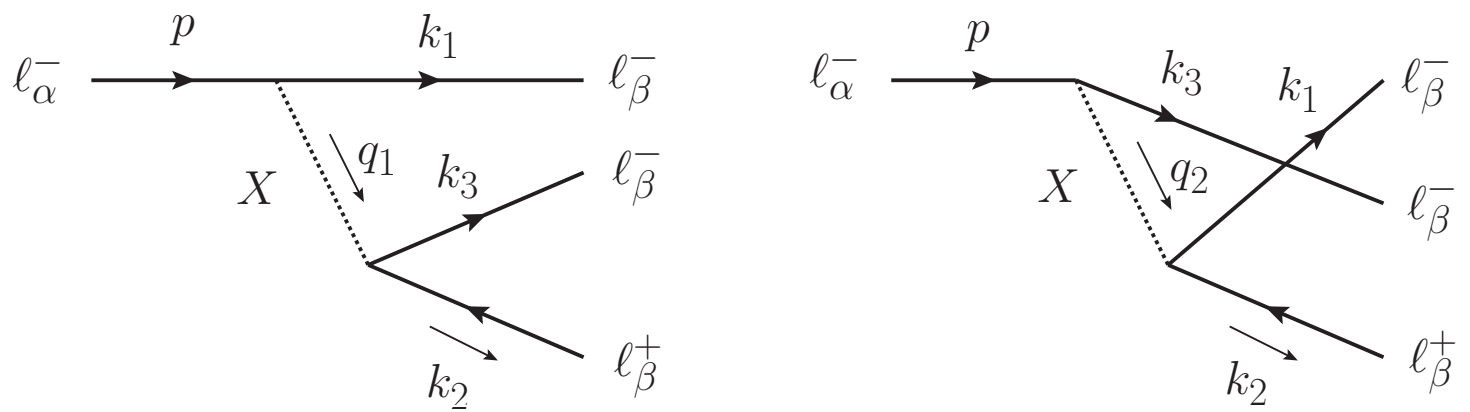

Figure 11. Feynman diagrams of the process $\ell_{\alpha}^{-} \rightarrow \ell_{\beta}^{-} \ell_{\beta}^{-} \ell_{\beta}^{+}$, in which $\ell_{\alpha}^{-}$represents the SM charged lepton and $X=\phi, Z^{\prime}, A^{\prime}$.

where $s_{12}=\left(p-k_{3}\right)^{2}$ and $s_{23}=\left(p-k_{1}\right)^{2}$. The integral ranges of $s_{12}$ and $s_{23}$ are given by

$$
\begin{aligned}
s_{23}^{\max } & =\left(\hat{E}_{2}+\hat{E}_{3}\right)^{2}-\left(\hat{P}_{2}-\hat{P}_{3}\right)^{2}, \\
s_{23}^{\min } & =\left(\hat{E}_{2}+\hat{E}_{3}\right)^{2}-\left(\hat{P}_{2}+\hat{P}_{3}\right)^{2}, \\
s_{12}^{\max } & =\left(m_{\alpha}-m_{\beta}\right)^{2}, \\
s_{12}^{\min } & =4 m_{\beta}^{2},
\end{aligned}
$$

where

$$
\begin{aligned}
& \hat{E}_{2}=\frac{1}{2 \sqrt{s_{12}}}\left(m_{\alpha}^{2}-s_{12}-m_{\beta}^{2}\right), \\
& \hat{E}_{3}=\frac{\sqrt{s_{12}}}{2}, \\
& \hat{P}_{2}=\frac{1}{2} \sqrt{s_{12}-2\left(m_{\alpha}^{2}+m_{\beta}^{2}\right)+\frac{\left(m_{\alpha}^{2}-m_{\beta}^{2}\right)^{2}}{s_{12}}}, \\
& \hat{P}_{3}=\frac{1}{2} \sqrt{s_{12}-4 m_{\beta}^{2}} .
\end{aligned}
$$

The amplitude is written as

$$
\frac{1}{2} \sum_{\text {spin }}|\mathcal{M}|^{2}=\frac{C^{2}}{2}\left[P_{X}^{2}\left(s_{23}\right) M_{1}^{2}+P_{X}^{2}\left(s_{12}\right) M_{2}^{2}-2 P_{X}\left(s_{23}\right) P_{X}\left(s_{12}\right) \operatorname{Re}\left[M_{3}^{2}\right]\right],
$$

where $P_{X}\left(s_{23(12)}\right)=\left(s_{23(12)}-m_{X}^{2}\right)^{-1}, X=\phi, Z^{\prime}, A^{\prime}$, and $C=y_{e} y_{e \mu}^{\prime},\left(g^{\prime}\right)^{2} s^{3} c$ and $\mu_{e} \mu^{\prime}$ for the scalar, the vector and the dipole interaction, respectively. For the scalar interaction,

$$
\begin{aligned}
M_{1}^{2}= & -4\left(s_{23}-4 m_{\beta}^{2}\right)\left(s_{23}-\left(m_{\alpha}+m_{\beta}\right)^{2}\right) \\
M_{2}^{2}= & -4\left(s_{12}-4 m_{\beta}^{2}\right)\left(s_{12}-\left(m_{\alpha}+m_{\beta}\right)^{2}\right) \\
M_{3}^{2}= & 2 s_{12} s_{23}+4 m_{\beta}\left(m_{\alpha}+m_{\beta}\right)\left(s_{12}+s_{23}\right) \\
& \quad-2 m_{\beta}\left(m_{\alpha}+m_{\beta}\right)\left(5 m_{\beta}^{5}+2 m_{\alpha} m_{\beta}+m_{\alpha}^{2}\right),
\end{aligned}
$$


for the vector interaction,

$$
\begin{aligned}
M_{1}^{2}=- & 8\left\{2 s_{12}^{2}+s_{23}^{2}+2 s_{12} s_{23}-\left(m_{\alpha}-m_{\beta}\right)^{2} s_{23}-2\left(m_{\alpha}^{2}+3 m_{\beta}^{2}\right) s_{12}\right. \\
& \left.+2 m_{\beta}^{2}\left(m_{\alpha}+m_{\beta}\right)^{2}\right\}, \\
M_{2}^{2}=- & 8\left\{2 s_{23}^{2}+s_{12}^{2}+2 s_{12} s_{23}-\left(m_{\alpha}-m_{\beta}\right)^{2} s_{12}-2\left(m_{\alpha}^{2}+3 m_{\beta}^{2}\right) s_{23}\right. \\
& \left.+2 m_{\beta}^{2}\left(m_{\alpha}+m_{\beta}\right)^{2}\right\}, \\
M_{3}^{2}=8 & \left(s_{12}+s_{23}+m_{\beta}\left(m_{\alpha}+m_{\beta}\right)\right)\left(s_{12}+s_{23}-m_{\alpha}^{2}+m_{\alpha} m_{\beta}-2 m_{\beta}^{2}\right),
\end{aligned}
$$

and for the dipole interaction,

$$
\begin{aligned}
& M_{1}^{2}=-4 s_{23}\left\{s_{23}^{3}+4\left(s_{12}+s_{23}\right) s_{12} s_{23}-\left(m_{\alpha}-m_{\beta}\right)^{2} s_{23}^{2}\right. \\
& \left.-4\left(m_{\alpha}^{2}+3 m_{\beta}^{2}\right) s_{12} s_{23}+8 m_{\beta}^{2}\left(m_{\alpha}+m_{\beta}\right)^{2} s_{23}-4 m_{\beta}^{2}\left(m_{\alpha}^{2}-m_{\beta}^{2}\right)^{2}\right\}, \\
& M_{2}^{2}=-4 s_{12}\left\{s_{12}^{3}+4\left(s_{12}+s_{23}\right) s_{12} s_{23}-\left(m_{\alpha}-m_{\beta}\right)^{2} s_{12}^{2}\right. \\
& \left.-4\left(m_{\alpha}^{2}+3 m_{\beta}^{2}\right) s_{12} s_{23}+8 m_{\beta}^{2}\left(m_{\alpha}+m_{\beta}\right)^{2} s_{12}-4 m_{\beta}^{2}\left(m_{\alpha}^{2}-m_{\beta}^{2}\right)^{2}\right\}, \\
& M_{3}^{2}=2\left\{2\left(s_{12}^{2}+s_{23}^{2}\right) s_{12} s_{23}+5 s_{12}^{2} s_{23}^{2}-2\left(m_{\alpha}^{2}+3 m_{\beta}^{2}\right)\left(s_{12}+s_{23}\right) s_{12} s_{23}\right. \\
& +m_{\beta}\left(m_{\alpha}+m_{\beta}\right)\left(3 m_{\alpha}^{2}+2 m_{\alpha} m_{\beta}+11 m_{\beta}^{2}\right) s_{12} s_{23} \\
& \left.-2 m_{\beta}^{2}\left(m_{\alpha}^{2}-m_{\beta}^{2}\right)^{2}\left(s_{12}+s_{23}\right)-4 m_{\beta}^{3}\left(m_{\alpha}+m_{\beta}\right)\left(m_{\alpha}^{2}-m_{\beta}^{2}\right)^{2}\right\},
\end{aligned}
$$

where $m_{\alpha}$ stands for the mass of the $\alpha$ flavor charged lepton.

The partial width of the muon decay into an electron and a light boson is given by

$$
\begin{aligned}
\Gamma(\mu \rightarrow e \phi)= & \frac{\lambda_{e \mu}^{2}}{16 \pi} m_{\mu} \lambda\left(\frac{m_{e}^{2}}{m_{\mu}^{2}}, \frac{m_{\phi}^{2}}{m_{\mu}^{2}}\right)\left\{1+2 \frac{m_{e}}{m_{\mu}}+\frac{m_{e}^{2}-m_{\phi}^{2}}{m_{\mu}^{2}}\right\}, \\
\Gamma(\mu \rightarrow e a)= & \frac{c_{e \mu}^{2}}{16 \pi f_{a}^{2}} m_{\mu} \lambda\left(\frac{m_{e}^{2}}{m_{\mu}^{2}}, \frac{m_{a}^{2}}{m_{\mu}^{2}}\right)\left\{\left(1-\frac{m_{e}^{2}}{m_{\mu}^{2}}\right)^{2}-\left(1+\frac{m_{e}}{m_{\mu}}\right)^{2} \frac{m_{a}^{2}}{m_{\mu}^{2}}\right\}, \\
\Gamma\left(\mu \rightarrow e Z^{\prime}\right)= & \frac{\left(g^{\prime} s c\right)^{2}}{16 \pi} m_{\mu} \lambda\left(\frac{m_{e}^{2}}{m_{\mu}^{2}}, \frac{m_{Z^{\prime}}^{2}}{m_{\mu}^{2}}\right) \frac{m_{\mu}^{2}}{m_{Z^{\prime}}^{2}} \\
& \times\left\{\left(1-\frac{m_{e}^{2}}{m_{\mu}^{2}}\right)^{2}-\frac{m_{Z^{\prime}}^{4}}{m_{\mu}^{4}}+\left(1-6 \frac{m_{e}}{m_{\mu}}+\frac{m_{e}^{2}-m_{Z^{\prime}}^{2}}{m_{\mu}^{2}}\right) \frac{m_{Z^{\prime}}^{2}}{m_{\mu}^{2}}\right\}, \\
\Gamma\left(\mu \rightarrow e A^{\prime}\right)= & \frac{\left(\mu^{\prime} m_{\mu}\right)^{2}}{16 \pi} m_{\mu} \lambda\left(\frac{m_{e}^{2}}{m_{\mu}^{2}}, \frac{m_{A^{\prime}}^{2}}{m_{\mu}^{2}}\right) \\
& \times\left[2\left\{\left(1-\frac{m_{e}^{2}}{m_{\mu}^{2}}\right)^{2}-\frac{m_{A^{\prime}}^{4}}{m_{\mu}^{4}}\right\}-\left(1+6 \frac{m_{e}}{m_{\mu}}+\frac{m_{e}^{2}-m_{A^{\prime}}^{2}}{m_{\mu}^{2}}\right) \frac{m_{A^{\prime}}^{2}}{m_{\mu}^{2}}\right],
\end{aligned}
$$

for the scalar, pseudoscalar, vector, and dipole interaction, respectively, and $\lambda(a, b)$ is the Kallen function given in eq. (2.4).

Open Access. This article is distributed under the terms of the Creative Commons Attribution License (CC-BY 4.0), which permits any use, distribution and reproduction in any medium, provided the original author(s) and source are credited. 


\section{References}

[1] S. Baek, N.G. Deshpande, X.G. He and P. Ko, Muon anomalous $g-2$ and gauged $L_{\mu}-L_{\tau}$ models, Phys. Rev. D 64 (2001) 055006 [hep-ph/0104141] [InSPIRE].

[2] M. Pospelov, Secluded U(1) below the weak scale, Phys. Rev. D 80 (2009) 095002 [arXiv:0811.1030] [INSPIRE].

[3] W. Altmannshofer, S. Gori, M. Pospelov and I. Yavin, Neutrino Trident Production: A Powerful Probe of New Physics with Neutrino Beams, Phys. Rev. Lett. 113 (2014) 091801 [arXiv: 1406.2332] [INSPIRE].

[4] D. Tucker-Smith and N. Weiner, Inelastic dark matter, Phys. Rev. D 64 (2001) 043502 [hep-ph/0101138] [INSPIRE].

[5] C. Boehm and P. Fayet, Scalar dark matter candidates, Nucl. Phys. B 683 (2004) 219 [hep-ph/0305261] [INSPIRE].

[6] M. Pospelov, A. Ritz and M.B. Voloshin, Secluded WIMP Dark Matter, Phys. Lett. B 662 (2008) 53 [arXiv:0711.4866] [InSPIRE].

[7] E. Izaguirre, G. Krnjaic, P. Schuster and N. Toro, Analyzing the Discovery Potential for Light Dark Matter, Phys. Rev. Lett. 115 (2015) 251301 [arXiv:1505.00011] [INSPIRE].

[8] S. Knapen, T. Lin and K.M. Zurek, Light Dark Matter: Models and Constraints, Phys. Rev. D 96 (2017) 115021 [arXiv: 1709.07882] [INSPIRE].

[9] P.J. Fitzpatrick, H. Liu, T.R. Slatyer and Y.-D. Tsai, New Pathways to the Relic Abundance of Vector-Portal Dark Matter, arXiv:2011.01240 [INSPIRE].

[10] M. Duerr, T. Ferber, C. Garcia-Cely, C. Hearty and K. Schmidt-Hoberg, Long-lived Dark Higgs and Inelastic Dark Matter at Belle II, JHEP 04 (2021) 146 [arXiv:2012.08595] [INSPIRE].

[11] K. Asai, S. Okawa and K. Tsumura, Search for U(1) $L_{L_{\mu}-L_{\tau}}$ charged Dark Matter with neutrino telescope, JHEP 03 (2021) 047 [arXiv:2011.03165] [INSPIRE].

[12] M. Escudero, D. Hooper, G. Krnjaic and M. Pierre, Cosmology with A Very Light $L_{\mu}-L_{\tau}$ Gauge Boson, JHEP 03 (2019) 071 [arXiv: 1901.02010] [InSPIRE].

[13] M. Escudero and S.J. Witte, A CMB search for the neutrino mass mechanism and its relation to the Hubble tension, Eur. Phys. J. C 80 (2020) 294 [arXiv: 1909.04044] [INSPIRE].

[14] M. Escudero Abenza, Precision early universe thermodynamics made simple: $N_{\mathrm{eff}}$ and neutrino decoupling in the Standard Model and beyond, JCAP 05 (2020) 048 [arXiv:2001.04466] [INSPIRE].

[15] T. Araki et al., Resolving the Hubble tension in a $\mathrm{U}(1)_{L_{\mu}-L_{\tau}}$ model with Majoron, arXiv:2103.07167 [INSPIRE].

[16] K. Ioka and K. Murase, IceCube PeV-EeV neutrinos and secret interactions of neutrinos, Prog. Theor. Exp. Phys. 2014 (2014) 061E01 [arXiv: 1404.2279] [InSPIRE].

[17] K.C.Y. Ng and J.F. Beacom, Cosmic neutrino cascades from secret neutrino interactions, Phys. Rev. D 90 (2014) 065035 [Erratum ibid. 90 (2014) 089904] [arXiv:1404.2288] [INSPIRE].

[18] M. Ibe and K. Kaneta, Cosmic neutrino background absorption line in the neutrino spectrum at IceCube, Phys. Rev. D 90 (2014) 053011 [arXiv:1407.2848] [INSPIRE]. 
[19] T. Araki, F. Kaneko, Y. Konishi, T. Ota, J. Sato and T. Shimomura, Cosmic neutrino spectrum and the muon anomalous magnetic moment in the gauged $L_{\mu}-L_{\tau}$ model, Phys. Rev. D 91 (2015) 037301 [arXiv: 1409.4180] [INSPIRE].

[20] A. Kamada and H.-B. Yu, Coherent Propagation of PeV Neutrinos and the Dip in the Neutrino Spectrum at IceCube, Phys. Rev. D 92 (2015) 113004 [arXiv:1504.00711] [INSPIRE].

[21] A. DiFranzo and D. Hooper, Searching for MeV-Scale Gauge Bosons with IceCube, Phys. Rev. D 92 (2015) 095007 [arXiv:1507.03015] [InSPIRE].

[22] T. Araki, F. Kaneko, T. Ota, J. Sato and T. Shimomura, MeV scale leptonic force for cosmic neutrino spectrum and muon anomalous magnetic moment, Phys. Rev. D 93 (2016) 013014 [arXiv: 1508.07471] [INSPIRE].

[23] S. Mohanty, A. Narang and S. Sadhukhan, Cutoff of IceCube Neutrino Spectrum due to t-channel Resonant Absorption by $C \nu B, J C A P 03$ (2019) 041 [arXiv: 1808.01272] [INSPIRE].

[24] J.A. Carpio, K. Murase, I.M. Shoemaker and Z. Tabrizi, High-energy cosmic neutrinos as a probe of the vector mediator scenario in light of the muon $g-2$ anomaly and Hubble tension, arXiv:2104.15136 [INSPIRE].

[25] CHARM collaboration, Search for Axion Like Particle Production in $400 \mathrm{GeV}$ Proton-Copper Interactions, Phys. Lett. B 157 (1985) 458 [inSPIRE].

[26] A. Konaka et al., Search for Neutral Particles in Electron Beam Dump Experiment, Phys. Rev. Lett. 57 (1986) 659 [INSPIRE].

[27] E.M. Riordan et al., A Search for Short Lived Axions in an Electron Beam Dump Experiment, Phys. Rev. Lett. 59 (1987) 755 [INSPIRE].

[28] J.D. Bjorken et al., Search for Neutral Metastable Penetrating Particles Produced in the SLAC Beam Dump, Phys. Rev. D 38 (1988) 3375 [INSPIRE].

[29] A. Bross, M. Crisler, S.H. Pordes, J. Volk, S. Errede and J. Wrbanek, A Search for Shortlived Particles Produced in an Electron Beam Dump, Phys. Rev. Lett. 67 (1991) 2942 [InSPIRE].

[30] M. Davier and H. Nguyen Ngoc, An Unambiguous Search for a Light Higgs Boson, Phys. Lett. B 229 (1989) 150 [INSPIRE].

[31] S.N. Gninenko, Constraints on sub-GeV hidden sector gauge bosons from a search for heavy neutrino decays, Phys. Lett. B $\mathbf{7 1 3}$ (2012) 244 [arXiv:1204.3583] [INSPIRE].

[32] J.D. Bjorken, R. Essig, P. Schuster and N. Toro, New Fixed-Target Experiments to Search for Dark Gauge Forces, Phys. Rev. D 80 (2009) 075018 [arXiv:0906.0580] [InSPIRE].

[33] S. Andreas, C. Niebuhr and A. Ringwald, New Limits on Hidden Photons from Past Electron Beam Dumps, Phys. Rev. D 86 (2012) 095019 [arXiv:1209.6083] [INSPIRE].

[34] M. Bauer, P. Foldenauer and J. Jaeckel, Hunting All the Hidden Photons, JHEP 07 (2018) 094 [arXiv: 1803.05466] [INSPIRE].

[35] M. Fabbrichesi, E. Gabrielli and G. Lanfranchi, The Dark Photon, arXiv:2005.01515 [INSPIRE].

[36] M.W. Winkler, Decay and detection of a light scalar boson mixing with the Higgs boson, Phys. Rev. D 99 (2019) 015018 [arXiv:1809.01876] [InSPIRE].

[37] M. Bauer, M. Heiles, M. Neubert and A. Thamm, Axion-Like Particles at Future Colliders, Eur. Phys. J. C 79 (2019) 74 [arXiv:1808.10323] [inSPIRE]. 
[38] S. Kanemura, T. Moroi and T. Tanabe, Beam dump experiment at future electron-positron colliders, Phys. Lett. B $\mathbf{7 5 1}$ (2015) 25 [arXiv:1507.02809] [INSPIRE].

[39] Y. Sakaki and D. Ueda, Searching for new light particles at the international linear collider main beam dump, Phys. Rev. D 103 (2021) 035024 [arXiv: 2009.13790] [InSPIRE].

[40] K. Asai, T. Moroi and A. Niki, Leptophilic Gauge Bosons at ILC Beam Dump Experiment, Phys. Lett. B 818 (2021) 136374 [arXiv:2104.00888] [INSPIRE].

[41] K. Asai, S. Iwamoto, Y. Sakaki and D. Ueda, New physics searches at the ILC positron and electron beam dumps, arXiv:2105.13768 [INSPIRE].

[42] R. Harnik, J. Kopp and P.A.N. Machado, Exploring nu Signals in Dark Matter Detectors, JCAP 07 (2012) 026 [arXiv: 1202.6073] [INSPIRE].

[43] A. Caputo, C.A.J. O'Hare, A.J. Millar and E. Vitagliano, Dark photon limits: a cookbook, arXiv:2105.04565 [INSPIRE].

[44] D. Kazanas, R.N. Mohapatra, S. Nussinov, V.L. Teplitz and Y. Zhang, Supernova Bounds on the Dark Photon Using its Electromagnetic Decay, Nucl. Phys. B 890 (2014) 17 [arXiv: 1410.0221] [INSPIRE].

[45] D. Croon, G. Elor, R.K. Leane and S.D. McDermott, Supernova Muons: New Constraints on $Z^{\prime}$ Bosons, Axions and ALPs, JHEP 01 (2021) 107 [arXiv:2006.13942] [INSPIRE].

[46] J. Heeck, Lepton flavor violation with light vector bosons, Phys. Lett. B 758 (2016) 101 [arXiv: 1602.03810] [INSPIRE].

[47] W. Altmannshofer, C.-Y. Chen, P.S. Bhupal Dev and A. Soni, Lepton flavor violating Z' explanation of the muon anomalous magnetic moment, Phys. Lett. B 762 (2016) 389 [arXiv: 1607.06832] [INSPIRE].

[48] J. Heeck and W. Rodejohann, Lepton flavor violation with displaced vertices, Phys. Lett. B 776 (2018) 385 [arXiv:1710.02062] [INSPIRE].

[49] S. Iguro, Y. Omura and M. Takeuchi, Probing $\mu \tau$ flavor-violating solutions for the muon $g-2$ anomaly at Belle II, JHEP 09 (2020) 144 [arXiv:2002.12728] [INSPIRE].

[50] H. Davoudiasl, R. Marcarelli, N. Miesch and E.T. Neil, Searching for flavor-violating ALPs in Higgs boson decays, Phys. Rev. D 104 (2021) 055022 [arXiv:2105.05866] [INSPIRE].

[51] M. Bauer, M. Neubert, S. Renner, M. Schnubel and A. Thamm, Axionlike Particles, Lepton-Flavor Violation, and a New Explanation of $a_{\mu}$ and $a_{e}$, Phys. Rev. Lett. 124 (2020) 211803 [arXiv: 1908.00008] [INSPIRE].

[52] C. Cornella, P. Paradisi and O. Sumensari, Hunting for ALPs with Lepton Flavor Violation, JHEP 01 (2020) 158 [arXiv:1911.06279] [INSPIRE].

[53] M. Endo, S. Iguro and T. Kitahara, Probing e $\mu$ flavor-violating ALP at Belle II, JHEP 06 (2020) 040 [arXiv: 2002.05948] [INSPIRE].

[54] M. Lindner, M. Platscher and F.S. Queiroz, A Call for New Physics: The Muon Anomalous Magnetic Moment and Lepton Flavor Violation, Phys. Rept. 731 (2018) 1 [arXiv: 1610.06587] [INSPIRE].

[55] J.L. Diaz-Cruz and J.J. Toscano, Lepton flavor violating decays of Higgs bosons beyond the standard model, Phys. Rev. D 62 (2000) 116005 [hep-ph/9910233] [INSPIRE]. 
[56] E. Arganda, A.M. Curiel, M.J. Herrero and D. Temes, Lepton flavor violating Higgs boson decays from massive seesaw neutrinos, Phys. Rev. D 71 (2005) 035011 [hep-ph/0407302] [INSPIRE].

[57] R.D. Peccei and H.R. Quinn, CP Conservation in the Presence of Instantons, Phys. Rev. Lett. 38 (1977) 1440 [INSPIRE].

[58] S. Weinberg, A New Light Boson?, Phys. Rev. Lett. 40 (1978) 223 [InSPIRE].

[59] F. Wilczek, Problem of Strong $P$ and T Invariance in the Presence of Instantons, Phys. Rev. Lett. 40 (1978) 279 [INSPIRE].

[60] J. Preskill, M.B. Wise and F. Wilczek, Cosmology of the Invisible Axion, Phys. Lett. B 120 (1983) 127 [INSPIRE].

[61] L.F. Abbott and P. Sikivie, A Cosmological Bound on the Invisible Axion, Phys. Lett. B 120 (1983) 133 [INSPIRE].

[62] M. Dine and W. Fischler, The Not So Harmless Axion, Phys. Lett. B 120 (1983) 137 [INSPIRE].

[63] F. Wilczek, Axions and Family Symmetry Breaking, Phys. Rev. Lett. 49 (1982) 1549 [INSPIRE].

[64] A. Davidson and K.C. Wali, Minimal flavor unification via multigenerational Peccei-Quinn symmetry, Phys. Rev. Lett. 48 (1982) 11 [inSPIRE].

[65] Y. Ema, K. Hamaguchi, T. Moroi and K. Nakayama, Flaxion: a minimal extension to solve puzzles in the standard model, JHEP 01 (2017) 096 [arXiv:1612.05492] [INSPIRE].

[66] L. Calibbi, F. Goertz, D. Redigolo, R. Ziegler and J. Zupan, Minimal axion model from flavor, Phys. Rev. D 95 (2017) 095009 [arXiv: 1612.08040] [InSPIRE].

[67] Y. Ema, D. Hagihara, K. Hamaguchi, T. Moroi and K. Nakayama, Supersymmetric Flaxion, JHEP 04 (2018) 094 [arXiv: 1802. 07739] [INSPIRE].

[68] L. Calibbi, D. Redigolo, R. Ziegler and J. Zupan, Looking forward to Lepton-flavor-violating ALPs, JHEP 09 (2021) 173 [arXiv: 2006. 04795] [INSPIRE].

[69] R. Foot, New Physics From Electric Charge Quantization?, Mod. Phys. Lett. A 6 (1991) 527 [INSPIRE].

[70] X.G. He, G.C. Joshi, H. Lew and R.R. Volkas, New-Z' phenomenology, Phys. Rev. D 43 (1991) 22 [INSPIRE].

[71] R. Foot, X.G. He, H. Lew and R.R. Volkas, Model for a light $Z^{\prime}$ boson, Phys. Rev. D 50 (1994) 4571 [hep-ph/9401250] [INSPIRE].

[72] MEG collaboration, Search for the lepton flavour violating decay $\mu^{+} \rightarrow \mathrm{e}^{+} \gamma$ with the full dataset of the MEG experiment, Eur. Phys. J. C 76 (2016) 434 [arXiv:1605.05081] [INSPIRE].

[73] BABAR collaboration, Searches for Lepton Flavor Violation in the Decays $\tau^{ \pm} \rightarrow e^{ \pm} \gamma$ and $\tau^{ \pm} \rightarrow \mu^{ \pm} \gamma$, Phys. Rev. Lett. 104 (2010) 021802 [arXiv:0908.2381] [INSPIRE].

[74] T. Nomura, H. Okada and Y. Uesaka, A radiatively induced neutrino mass model with hidden local $\mathrm{U}(1)$ and $L F V$ processes $\ell_{i} \rightarrow \ell_{j} \gamma, \mu \rightarrow e Z^{\prime}$ and $\mu e \rightarrow e e$, JHEP 01 (2021) 016 [arXiv: 2005. 05527] [INSPIRE]. 
[75] C.F. von Weizsacker, Radiation emitted in collisions of very fast electrons, Z. Phys. 88 (1934) 612 [INSPIRE].

[76] E.J. Williams, Correlation of certain collision problems with radiation theory, Mat.-Fys. Medd. 13 (1935) 1.

[77] K.J. Kim and Y.-S. Tsai, Improved Weizsacker-Williams method and its application to lepton and W-boson pair production, Phys. Rev. D 8 (1973) 3109 [INSPIRE].

[78] Y.-S. Tsai, Axion bremsstrahlung by an electron beam, Phys. Rev. D 34 (1986) 1326 [INSPIRE].

[79] S. Andreas, Light Weakly Interacting Particles: Constraints and Connection to Dark Matter, Ph.D. Thesis, University of Hamburg, Hamburg Germany (2013) [https://doi.org/10.3204/DESY-THESIS-2013-024].

[80] Particle Data collaboration, Review of Particle Physics, Prog. Theor. Exp. Phys. 2020 (2020) 083C01 [inSPIRE].

[81] Y.-S. Liu, D. McKeen and G.A. Miller, Validity of the Weizsäcker-Williams approximation and the analysis of beam dump experiments: Production of a new scalar boson, Phys. Rev. D 95 (2017) 036010 [arXiv: 1609.06781] [INSPIRE].

[82] SINDRUM collaboration, Search for the Decay $\mu^{+} \rightarrow e^{+} e^{+} e^{-}$, Nucl. Phys. B 299 (1988) 1 [INSPIRE].

[83] TWIST collaboration, Search for two body muon decay signals, Phys. Rev. D 91 (2015) 052020 [arXiv: 1409.0638] [INSPIRE].

[84] PIENU collaboration, Improved search for two body muon decay $\mu^{+} \rightarrow e^{+} X_{H}$, Phys. Rev. $D$ 101 (2020) 052014 [arXiv:2002.09170] [InSPIRE].

[85] S.E. Derenzo, Measurement of the low-energy end of the mu-plus decay spectrum, Phys. Rev. 181 (1969) 1854 [INSPIRE].

[86] R. Essig, R. Harnik, J. Kaplan and N. Toro, Discovering New Light States at Neutrino Experiments, Phys. Rev. D 82 (2010) 113008 [arXiv:1008.0636] [InSPIRE].

[87] Y.-S. Liu and G.A. Miller, Validity of the Weizsäcker-Williams approximation and the analysis of beam dump experiments: Production of an axion, a dark photon, or a new axial-vector boson, Phys. Rev. D 96 (2017) 016004 [arXiv:1705. 01633] [INSPIRE].

[88] J.L. Feng, I. Galon, F. Kling and S. Trojanowski, ForwArd Search ExpeRiment at the LHC, Phys. Rev. D 97 (2018) 035001 [arXiv:1708.09389] [INSPIRE].

[89] J.L. Feng, I. Galon, F. Kling and S. Trojanowski, Dark Higgs bosons at the ForwArd Search ExpeRiment, Phys. Rev. D 97 (2018) 055034 [arXiv:1710.09387] [INSPIRE].

[90] FASER collaboration, FASER's physics reach for long-lived particles, Phys. Rev. D 99 (2019) 095011 [arXiv:1811.12522] [INSPIRE].

[91] T. Araki, K. Asai, H. Otono, T. Shimomura and Y. Takubo, work in progress. 